\title{
Phantom Ideals and Cotorsion Pairs in Extriangulated Categories
}

\author{
Tiwei Zhao and Zhaoyong Huang*
}

\begin{abstract}
In this paper, we introduce and study relative phantom morphisms in extriangulated categories defined by Nakaoka and Palu. Then using their properties, we show that if $(\mathscr{C}, \mathbb{E}, \mathfrak{s})$ is an extriangulated category with enough injective objects and projective objects, then there exists a bijective correspondence between any two of the following classes: (1) special precovering ideals of $\mathscr{C}$; $(2)$ special preenveloping ideals of $\mathscr{C}$; (3) additive subfunctors of $\mathbb{E}$ having enough special injective morphisms; and (4) additive subfunctors of $\mathbb{E}$ having enough special projective morphisms. Moreover, we show that if $(\mathscr{C}, \mathbb{E}, \mathfrak{s})$ is an extriangulated category with enough injective objects and projective morphisms, then there exists a bijective correspondence between the following two classes: (1) all object-special precovering ideals of $\mathscr{C}$; (2) all additive subfunctors of $\mathbb{E}$ having enough special injective objects.
\end{abstract}

\section{Introduction}

In algebra, geometry and topology, exact categories and triangulated categories are two fundamental structures. The interest of exact categories is manifold in many branches of mathematics, for example, in the categories of locally convex modules over a topological group [7], locally compact abelian groups [16], Banach modules over Banach algebras [17 and algebraic $K$-theory [26, 28], and so on. Triangulated categories were introduced in the mid 1960's by Verdier [30] in his thesis. Having their origins in algebraic geometry and algebraic topology 3, 11, 13], triangulated categories have also become indispensable in many different areas of mathematics by now. As expected, exact categories and triangulated categories are not independent of each other. A well-known fact is that triangulated categories which at the same time are abelian must be semisimple [22]. Also, there are a series of ways to produce triangulated categories from abelian ones, such as, taking the stable categories of Frobenius exact categories [12, or taking the homotopy categories or derived categories of complexes over abelian categories [22]. On the other hand, because of the recent development of the cluster theory, it becomes possible to produce abelian

Received August 16, 2017; Accepted May 20, 2018.

Communicated by Ching Hung Lam.

2010 Mathematics Subject Classification. 18G25, 16E30, 18E40.

Key words and phrases. phantom ideals, cotorsion pairs, extriangulated categories, (co)phantom morphisms, special precovering ideals, special preenveloping ideals.

${ }^{*}$ Corresponding author. 
categories from triangulated ones, that is, starting from a cluster category and taking a cluster tilting subcategory, one can get a suitable quotient category, which turns out to be abelian [19,23. In addition, exact categories and triangulated categories possess same properties in many homological invariants, for example in the aspect of approximation theory 1, 20,23]. Approximation theory is the main part of relative homological algebra and representation theory of algebras, and its starting point is to approximate arbitrary objects by a class of suitable subcategories. In this process, the notion of cotorsion pairs [27] provides a fruitful context, in particular, it is closely related to many important homological structures, for example, $t$-structure, co-t-structure, cluster tilting subcategories, and so on. In general, to transfer the homological properties between exact categories and triangulated categories, one needs to specify to the case of stable categories of Frobenius exact categories, and then lift (or descend) the associated definitions and statements, and finally adapt the proof so that it can apply to any exact (or triangulated) categories. However, it is not easy to do it in general case, especially in the third step. To overcome the difficulty, Nakaoka and Palu [24] introduced the notion of externally triangulated categories (extriangulated categories for short) by a careful looking what is necessary in the definition of cotorsion pairs in exact and triangulated cases. Under this notion, exact categories with a suitable assumption and extension-closed subcategories of triangulated categories both are externally triangulated (see Example 2.8), and hence, in some levels, it becomes easy to give uniform statements and proofs for the exact and triangulated settings 24,31 .

In an abstract category, objects and morphisms are two essential components; and by a well-known embedding from a category to its morphism category, objects can be viewed as special morphisms. In the classical approximation theory, we mainly concern the objects and the associated subcategories. However, in general case, it seems that the morphisms and the associated ideals also should be concerned in the approximation theory. From this point of view, Fu, Guil Asensio, Herzog and Torrecillas in 10 introduced the notion of ideal cotorsion pairs and developed the ideal approximation theory of exact categories. Inside it, the phantom ideal plays an important role in the aspect of providing a certain ideal cotorsion pair; and it has been investigated in algebraic topology [21], stable homotopy categories of spectra [2], triangulated categories [8, 25], and stable categories of finite group rings [4 6]. In particular, Herzog generalized in [15] the phantom morphism to the category of left $R$-modules of arbitrary associative ring $R$ in the following way: a morphism $f: M \rightarrow N$ of left $R$-modules is called a phantom morphism if the natural transformation $\operatorname{Tor}_{1}^{R}(-, f): \operatorname{Tor}_{1}^{R}(-, M) \rightarrow \operatorname{Tor}_{1}^{R}(-, N)$ is zero, or equivalently, the pullback of any short exact sequence along $f$ is pure exact. Then he showed that every module admits a phantom cover. As a generalization of the (classical) approximation theory for subcategories, Fu et 
al. developed in [10] the approximation theory of an exact category $\mathscr{A}$ for ideal cotorsion pairs. A careful look reveals that the essentially necessary matters in [10] are pullbacks and pushouts, that is, some special operations of functors. So this inspires us to establish the approximation theory in an additive category equipped with an additive bifunctor; in particular, we consider it in extriangulated categories, which not only unifies the ideal approximation theory in exact categories and triangulated categories, but also extends this theory to those categories which are neither exact nor triangulated as much as possible.

This paper is organized as follows.

In Section 2, we give some terminology and some preliminary results.

In Section 3, we first introduce the notion of relative phantom morphisms in an additive category, and then extend it to an extriangulated category. We study the relationship between relative phantom morphisms and relative injective morphisms, and give a sufficient condition such that they form a relative cotorsion pair.

In Section 4, we mainly discuss the role of phantom operations, and use it to investigate the interplay among special precovering ideals, special preenveloping ideals, additive subfunctors having enough special injective morphisms, and additive subfunctors having enough special projective morphisms. We show that if $(\mathscr{C}, \mathbb{E}, \mathfrak{s})$ is an extriangulated category with enough injective objects and projective objects, then we have the following bijective correspondences.

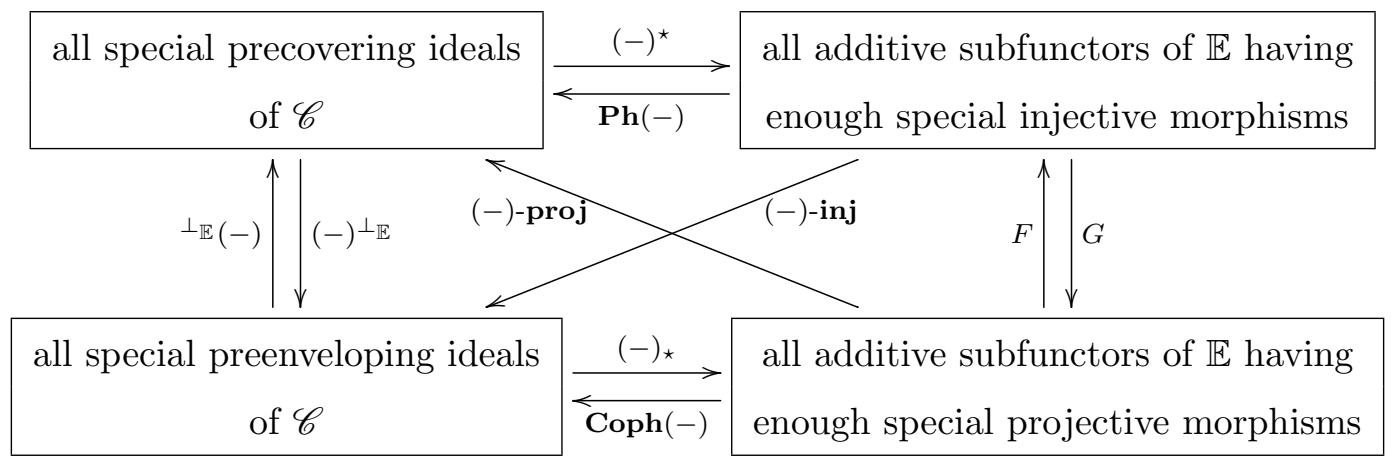

Here $F=(-)^{\star} \circ{ }^{\perp_{\mathbb{E}}}(-) \circ \mathbf{C o p h}(-)$ and $G=(-)_{\star} \circ(-)^{\perp_{\mathbb{E}}} \circ \mathbf{P h}(-)$, see Section 3 for the definitions of these functors.

In Section 5, we consider object-special precovering ideals, and show that if $(\mathscr{C}, \mathbb{E}, \mathfrak{s})$ is an extriangulated category with enough injective objects and projective morphisms, then we have the following bijective correspondence.

\begin{tabular}{|c|c|c|}
\hline $\begin{array}{c}\text { all object-special precovering ideals } \\
\text { of } \mathscr{C}\end{array}$ & $\stackrel{(-)^{\star}}{<} \begin{array}{c}\text { all additive subfunctors of } \mathbb{E} \text { having } \\
\text { enough special injective objects }\end{array}$ \\
\hline
\end{tabular}




\section{Preliminaries}

Throughout this paper, $\mathscr{C}$ is an additive category and $\mathbb{E}: \mathscr{C}^{\mathrm{op}} \times \mathscr{C} \rightarrow \mathfrak{A} b$ is a biadditive functor, where $\mathfrak{A} b$ is the category of abelian groups.

\subsection{E-extensions}

Definition 2.1. [24, Definitions 2.1 and 2.5] For any $A, C \in \mathscr{C}$, there is a corresponding abelian group $\mathbb{E}(C, A)$.

(1) An element $\delta \in \mathbb{E}(C, A)$ is called an $\mathbb{E}$-extension. More formally, an $\mathbb{E}$-extension is a triple $(A, \delta, C)$.

(2) The zero element 0 in $\mathbb{E}(C, A)$ is called the split $\mathbb{E}$-extension.

Let $a \in \mathscr{C}\left(A, A^{\prime}\right)$ and $c \in \mathscr{C}\left(C^{\prime}, C\right)$. Then we have the following commutative diagram

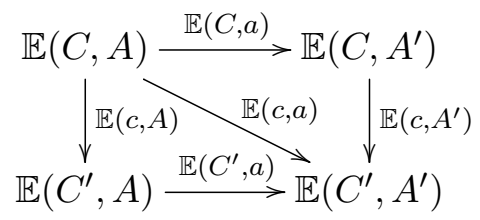

in $\mathfrak{A} b$. For an $\mathbb{E}$-extension $(A, \delta, C)$, we briefly write $a_{\star} \delta:=\mathbb{E}(C, a)(\delta)$ and $c^{\star} \delta:=$ $\mathbb{E}(c, A)(\delta)$. Then

$$
\mathbb{E}(c, a)(\delta)=c^{\star} a_{\star} \delta=a_{\star} c^{\star} \delta
$$

Definition 2.2. 24, Definition 2.3] Given two $\mathbb{E}$-extensions $(A, \delta, C)$ and $\left(A^{\prime}, \delta^{\prime}, C^{\prime}\right)$. A morphism from $\delta$ to $\delta^{\prime}$ is a pair $(a, c)$ of morphisms, where $a \in \mathscr{C}\left(A, A^{\prime}\right)$ and $c \in \mathscr{C}\left(C, C^{\prime}\right)$, such that $a_{\star} \delta=c^{\star} \delta$. In this case, we denote it by $(a, c): \delta \rightarrow \delta^{\prime}$.

Now let $A, C \in \mathscr{C}$. Two sequences of morphisms

$$
A \stackrel{x}{\rightarrow} B \stackrel{y}{\rightarrow} C \quad \text { and } \quad A \stackrel{x^{\prime}}{\rightarrow} B^{\prime} \stackrel{y^{\prime}}{\rightarrow} C
$$

are said to be equivalent if there exists an isomorphism $b \in \mathscr{C}\left(B, B^{\prime}\right)$ such that the following diagram

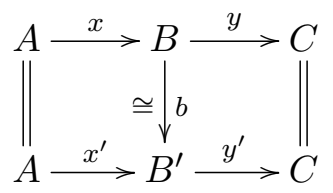

commutes. We denote by $[A \stackrel{x}{\rightarrow} B \stackrel{y}{\rightarrow} C]$ the equivalence class of $A \stackrel{x}{\rightarrow} B \stackrel{y}{\rightarrow} C$. In particular, we write $0:=\left[A \stackrel{\left(\begin{array}{l}1 \\ 0\end{array}\right)}{\longrightarrow} A \oplus C \stackrel{\left(\begin{array}{ll}0 & 1\end{array}\right)}{\rightarrow} C\right]$. 
Note that, for any pair $\delta \in \mathbb{E}(C, A)$ and $\delta^{\prime} \in \mathbb{E}\left(C^{\prime}, A^{\prime}\right)$, since $\mathbb{E}$ is biadditive, there exists a natural isomorphism

$$
\mathbb{E}\left(C \oplus C^{\prime}, A \oplus A^{\prime}\right) \cong \mathbb{E}(C, A) \oplus \mathbb{E}\left(C, A^{\prime}\right) \oplus \mathbb{E}\left(C^{\prime}, A\right) \oplus \mathbb{E}\left(C^{\prime}, A^{\prime}\right)
$$

We define the symbol $\delta \oplus \delta^{\prime}$ to be the element in $\mathbb{E}\left(C \oplus C^{\prime}, A \oplus A^{\prime}\right)$ corresponding to the element $\left(\delta, 0,0, \delta^{\prime}\right)$ in $\mathbb{E}(C, A) \oplus \mathbb{E}\left(C, A^{\prime}\right) \oplus \mathbb{E}\left(C^{\prime}, A\right) \oplus \mathbb{E}\left(C^{\prime}, A^{\prime}\right)$ through the above isomorphism.

Definition 2.3. [24, Definition 2.9] Let $\mathfrak{s}$ be a correspondence which associates an equivalence class $\mathfrak{s}(\delta)=[A \stackrel{x}{\rightarrow} B \stackrel{y}{\rightarrow} C]$ to each $\mathbb{E}$-extension $\delta \in \mathbb{E}(C, A)$. The $\mathfrak{s}$ is called a realization of $\mathbb{E}$ provided that it satisfies the following condition.

(R) Let $\delta \in \mathbb{E}(C, A)$ and $\delta^{\prime} \in \mathbb{E}\left(C^{\prime}, A^{\prime}\right)$ be any pair of $\mathbb{E}$-extensions with

$$
\mathfrak{s}(\delta)=[A \stackrel{x}{\rightarrow} B \stackrel{y}{\rightarrow} C] \quad \text { and } \quad \mathfrak{s}\left(\delta^{\prime}\right)=\left[A^{\prime} \stackrel{x^{\prime}}{\rightarrow} B^{\prime} \stackrel{y^{\prime}}{\rightarrow} C^{\prime}\right] .
$$

Then for any morphism $(a, c): \delta \rightarrow \delta^{\prime}$, there exists $b \in \mathscr{C}\left(B, B^{\prime}\right)$ such that the following diagram

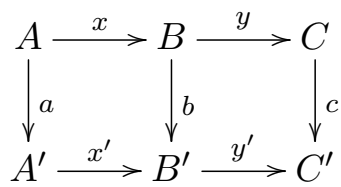

commutes.

Let $\mathfrak{s}$ be a realization of $\mathbb{E}$. If $\mathfrak{s}(\delta)=[A \stackrel{x}{\rightarrow} B \stackrel{y}{\rightarrow} C]$ for some $\mathbb{E}$-extension $\delta \in \mathbb{E}(C, A)$, then we say that the sequence $A \stackrel{x}{\rightarrow} B \stackrel{y}{\rightarrow} C$ realizes $\delta$; and in the condition $(\mathrm{R})$, we say that the triple $(a, b, c)$ realizes the morphism $(a, c)$.

Remark 2.4. Let $\mathfrak{s}$ be a realization of $\mathbb{E}$, and let $\delta \in \mathbb{E}(C, A)$ be an $\mathbb{E}$-extension with $\mathfrak{s}(\delta)=[A \stackrel{x}{\rightarrow} B \stackrel{y}{\rightarrow} C]$.

(1) For any $a \in \mathscr{C}\left(A, A^{\prime}\right)$, since $a_{\star} \delta=\operatorname{id}_{C}{ }^{\star} a_{\star} \delta$, there exists a morphism $\left(a, \operatorname{id}_{C}\right): \delta \rightarrow$ $a_{\star} \delta$. Assume that

$$
\mathfrak{s}\left(a_{\star} \delta\right)=\left[A^{\prime} \stackrel{x^{\prime}}{\longrightarrow} B^{\prime} \stackrel{y^{\prime}}{\rightarrow} C\right] .
$$

Then by the condition $(\mathrm{R})$, there exists a commutative diagram

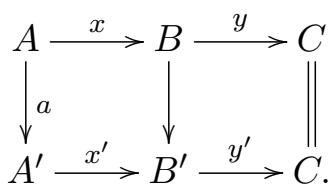


(2) For each $c \in \mathscr{C}\left(C^{\prime}, C\right)$, since $\operatorname{id}_{A \star} c^{\star} \delta=c^{\star} \delta$, there exists a morphism $\left(\operatorname{id}_{A}, c\right): c^{\star} \delta \rightarrow$ $\delta$. Assume that

$$
\mathfrak{s}\left(c^{\star} \delta\right)=\left[A \stackrel{x^{\prime \prime}}{\rightarrow} B^{\prime \prime} \stackrel{y^{\prime \prime}}{\rightarrow} C^{\prime}\right] .
$$

Then by the condition $(\mathrm{R})$, there exists a commutative diagram

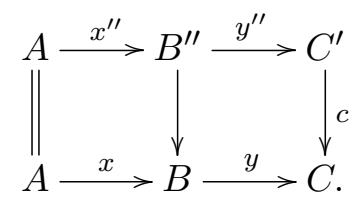

For any two equivalence classes $\left[A \stackrel{x}{\rightarrow} B \stackrel{y}{\rightarrow} C\right.$ ] and $\left[A^{\prime} \stackrel{x^{\prime}}{\rightarrow} B^{\prime} \stackrel{y^{\prime}}{\rightarrow} C^{\prime}\right]$, we define

$$
[A \stackrel{x}{\longrightarrow} B \stackrel{y}{\longrightarrow} C] \oplus\left[A^{\prime} \stackrel{x^{\prime}}{\longrightarrow} B^{\prime} \stackrel{y^{\prime}}{\longrightarrow} C^{\prime}\right]:=\left[A \oplus A^{\prime} \stackrel{x \oplus x^{\prime}}{\longrightarrow} B \oplus B^{\prime} \stackrel{y \oplus y^{\prime}}{\longrightarrow} C \oplus C^{\prime}\right] .
$$

Definition 2.5. 24, Definition 2.10] A realization $\mathfrak{s}$ of $\mathbb{E}$ is called additive if it satisfies the following conditions.

(1) For any $A, C \in \mathscr{C}$, the split $\mathbb{E}$-extension $0 \in \mathbb{E}(C, A)$ satisfies $\mathfrak{s}(0)=0$.

(2) For any pair of $\mathbb{E}$-extensions $\delta \in \mathbb{E}(C, A)$ and $\delta^{\prime} \in \mathbb{E}\left(C^{\prime}, A^{\prime}\right)$, we have $\mathfrak{s}\left(\delta \oplus \delta^{\prime}\right)=$ $\mathfrak{s}(\delta) \oplus \mathfrak{s}\left(\delta^{\prime}\right)$.

Let $\mathfrak{s}$ be an additive realization of $\mathbb{E}$. By [24, Remark 2.11], we have that if the sequence $A \stackrel{x}{\rightarrow} B \stackrel{y}{\rightarrow} C$ realizes 0 in $\mathbb{E}(C, A)$, then $x$ is a section and $y$ is a retraction.

\subsection{Externally triangulated categories}

Definition 2.6. [24, Definition 2.12] Let $\mathscr{C}$ be an additive category. We call the triple $(\mathscr{C}, \mathbb{E}, \mathfrak{s})$ an externally triangulated category (or extriangulated category for short) if it satisfies the following conditions.

(ET1) $\mathbb{E}: \mathscr{C}^{\mathrm{op}} \times \mathscr{C} \rightarrow \mathfrak{A} b$ is a biadditive functor.

$(\mathrm{ET} 2) \mathfrak{s}$ is an additive realization of $\mathbb{E}$.

(ET3) Let $\delta \in \mathbb{E}(C, A)$ and $\delta^{\prime} \in \mathbb{E}\left(C^{\prime}, A^{\prime}\right)$ be any pair of $\mathbb{E}$-extensions with

$$
\mathfrak{s}(\delta)=[A \stackrel{x}{\rightarrow} B \stackrel{y}{\rightarrow} C] \text { and } \mathfrak{s}\left(\delta^{\prime}\right)=\left[A^{\prime} \stackrel{x^{\prime}}{\rightarrow} B^{\prime} \stackrel{y^{\prime}}{\rightarrow} C^{\prime}\right] .
$$

For any commutative diagram

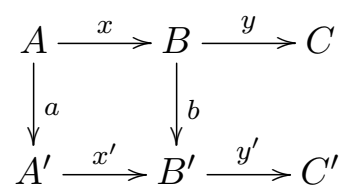

in $\mathscr{C}$, there exists a morphism $(a, c): \delta \rightarrow \delta^{\prime}$ which is realized by the triple $(a, b, c)$. 


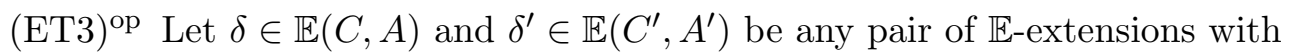

$$
\mathfrak{s}(\delta)=[A \stackrel{x}{\rightarrow} B \stackrel{y}{\rightarrow} C] \text { and } \mathfrak{s}\left(\delta^{\prime}\right)=\left[A^{\prime} \stackrel{x^{\prime}}{\rightarrow} B^{\prime} \stackrel{y^{\prime}}{\rightarrow} C^{\prime}\right] .
$$

For any commutative diagram

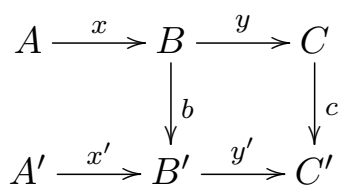

in $\mathscr{C}$, there exists a morphism $(a, c): \delta \rightarrow \delta^{\prime}$ which is realized by the triple $(a, b, c)$.

(ET4) Let $\delta \in \mathbb{E}(C, A)$ and $\rho \in \mathbb{E}(F, B)$ be any pair of $\mathbb{E}$-extensions with

$$
\mathfrak{s}(\delta)=[A \stackrel{x}{\rightarrow} B \stackrel{y}{\rightarrow} C] \text { and } \mathfrak{s}(\rho)=[B \stackrel{u}{\longrightarrow} D \stackrel{v}{\longrightarrow} F] .
$$

Then there exist an object $E \in \mathscr{C}$, an $\mathbb{E}$-extension $\xi$ with $\mathfrak{s}(\xi)=[A \stackrel{z}{\longrightarrow} D \stackrel{w}{\longrightarrow} E]$, and a commutative diagram

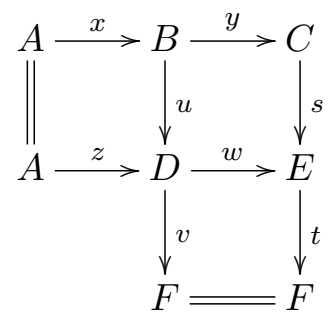

in $\mathscr{C}$, which satisfy the following compatibilities.

(i) $\mathfrak{s}\left(y_{\star} \rho\right)=[C \stackrel{s}{\rightarrow} E \stackrel{t}{\rightarrow} F]$.

(ii) $s^{\star} \xi=\delta$.

(iii) $x_{\star} \xi=t^{\star} \rho$.

(ET4) $)^{\text {op }}$ Let $\eta \in \mathbb{E}(E, A)$ and $\xi \in \mathbb{E}(F, C)$ be any pair of $\mathbb{E}$-extensions with

$$
\mathfrak{s}(\eta)=[A \stackrel{z}{\longrightarrow} D \stackrel{w}{\longrightarrow} E] \text { and } \mathfrak{s}(\xi)=[C \stackrel{s}{\longrightarrow} E \stackrel{t}{\longrightarrow} F] .
$$

Then there exist an object $B \in \mathscr{C}$, an $\mathbb{E}$-extension $\theta$ with $\mathfrak{s}(\theta)=[B \stackrel{u}{\rightarrow} D \stackrel{v}{\rightarrow} F]$, and a commutative diagram

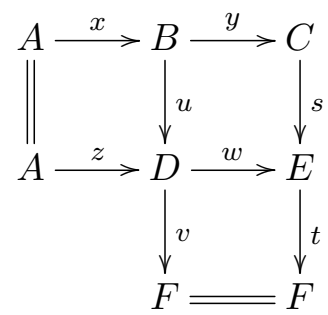

in $\mathscr{C}$ satisfying the following compatibilities. 

(i) $\mathfrak{s}\left(s^{\star} \eta\right)=[A \stackrel{x}{\longrightarrow} B \stackrel{y}{\rightarrow} C]$.
(ii) $y_{\star} \theta=\xi$.
(iii) $x_{\star} \eta=t^{\star} \theta$.

Definition 2.7. [24, Definition 2.19] Let $(\mathscr{C}, \mathbb{E}, \mathfrak{s})$ be a triple satisfying (ET1) and (ET2).

(1) If a sequence $A \stackrel{x}{\rightarrow} B \stackrel{y}{\rightarrow} C$ realizes an $\mathbb{E}$-extension $\delta \in \mathbb{E}(C, A)$, then we call the pair $(A \stackrel{x}{\rightarrow} B \stackrel{y}{\rightarrow} C, \delta)$ an $\mathbb{E}$-triangle, and write it in the following way

$$
A \stackrel{x}{\longrightarrow} B \stackrel{y}{\rightarrow} C \stackrel{\delta}{\rightarrow}
$$

In this case, $x$ is called an $\mathbb{E}$-inflation, and $y$ is called an $\mathbb{E}$-deflation.

(2) Let $A \stackrel{x}{\rightarrow} B \stackrel{y}{\rightarrow} C \stackrel{\delta}{\rightarrow}$ and $A^{\prime} \stackrel{x^{\prime}}{\rightarrow} B^{\prime} \stackrel{y^{\prime}}{\rightarrow} C^{\prime} \stackrel{\delta^{\prime}}{\rightarrow}$ be any pair of $\mathbb{E}$-triangles. If a triple $(a, b, c)$ realizes $(a, c): \delta \rightarrow \delta^{\prime}$ as in the condition $(\mathrm{R})$, then we write it as

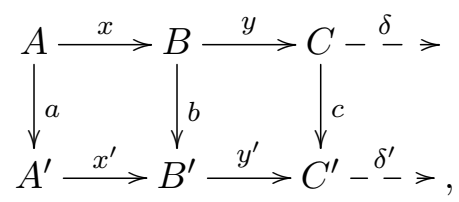

and call the triple $(a, b, c)$ a morphism of $\mathbb{E}$-triangles.

We collect some examples of extriangulated categories as follows.

Example 2.8. (1) All abelian categories, which are skeletally small or have enough projectives or injectives, are extriangulated categories. In fact, let $\mathcal{A}$ be such an abelian category. Then $\mathbb{E}:=\operatorname{Ext}_{\mathcal{A}}^{1}(-,-): \mathcal{A}^{\mathrm{op}} \times \mathcal{A} \rightarrow \mathfrak{A} \mathfrak{b}$ and the realization $\mathfrak{s}$ is defined by associating equivalence classes of short exact sequences to itself.

(2) Every closed additive subbifunctor $\mathcal{F}(-,-) \subseteq \operatorname{Ext}_{\mathcal{A}}^{1}(-,-)$ over an abelian category $\mathcal{A}$ such as in (1) induces an extriangulated category, where $\mathbb{E}:=\mathcal{F}(-,-)$ and its corresponding realization $\mathfrak{s}:=\left.\mathfrak{s}\right|_{\mathcal{F}}$, see [14, Definition 3.9, Lemma 3.13 and Proposition 3.14] for details. A trivial example is $\mathcal{F}=0$, that is, consider all split short exact sequences in $\mathcal{A}$. Moreover, for example, let $R$ be a ring, recall that a left $R$-module $M$ is called Gorenstein projective (see 9]) if there exists an exact sequence

$$
\cdots \rightarrow P_{1} \rightarrow P_{0} \rightarrow P^{0} \rightarrow P^{1} \rightarrow \cdots
$$

in $R$-Mod (the category of left $R$-modules) with all $P_{i}, P^{i}$ projective, such that it stays exact after applying the functor $\operatorname{Hom}_{R}(-, P)$ for any projective left $R$-module $P$, and $M=\operatorname{Im}\left(P_{0} \rightarrow P^{0}\right)$. Dually, the notion of Gorenstein injective left $R$-modules is defined. If moreover $R$ is a Gorenstein ring, that is, $R$ is a left and right Noetherian ring with finite 
left and right self-injective dimensions, then we may get the corresponding Gorenstein derived functor $\operatorname{GExt}_{R}^{1}(-,-)$ (see $[9]$ ). In this case, we have an extriangulated category $(R$-Mod, GExt, $\mathfrak{s})$, where the GExt-triangles are those short exact sequences in $R$-Mod which stay exact after applying the functor $\operatorname{Hom}_{R}(-, G)$ for any $G \in \mathcal{G P}$ (or equivalently, after applying the functor $\operatorname{Hom}_{R}(H,-)$ for any $\left.H \in \mathcal{G I}\right)$. Here $\mathcal{G P}$ and $\mathcal{G} \mathcal{I}$ stand for the full subcategories of $R$-Mod consisting of all Gorenstein projective and injective left $R$-modules respectively.

Recall that a short exact sequence $0 \rightarrow A \rightarrow B \rightarrow C \rightarrow 0$ in $R$-Mod is called pure exact if for any finitely presented left $R$-modules $F$, the induced sequence $\operatorname{Hom}_{R}(F, B) \rightarrow$ $\operatorname{Hom}_{R}(F, C) \rightarrow 0$ is exact. The pure injective (resp. pure projective) left $R$-modules are those modules which are injective (resp. projective) with respect to all short pure exact sequences in $R$-Mod. It is well known that there exist enough pure injective and pure projective objects in $R$-Mod. Following the corresponding pure projective and pure injective resolutions, we have the cohomological functor $\operatorname{PExt}_{R}^{1}(-,-)$ (see 18,29$\left.]\right)$. Then $(R$-Mod, PExt, $\mathfrak{s})$ is an extriangulated category, where the PExt-triangles are those short exact sequences in $R$-Mod which are pure exact.

(3) Exact categories $\mathcal{C}$, which are skeletally small or have enough projectives or injectives, are extriangulated categories, see [24, Example 2.13]. Note that for a ring $R$, the subcategory $\mathcal{G P}$ of $R$-Mod is closed under extensions and hence it is in fact an exact category. Thus we also have an extriangulated category $(\mathcal{G P}, \mathcal{E}, \mathfrak{s})$, where $\mathcal{E}$ is the collection of all short exact sequences in $R$-Mod whose terms are in $\mathcal{G P}$.

(4) Triangulated categories are extriangulated categories. In details, let $\mathcal{T}$ be a triangulated category and [1] the shift functor. Set $\mathbb{E}:=\mathcal{T}(-,-[1])$, and for any $\delta \in \mathbb{E}(Z, X)=$ $\mathcal{T}(Z, X[1])$, choose a triangle $X \rightarrow Y \rightarrow Z \stackrel{\delta}{\rightarrow} X[1]$ and define $\mathfrak{s}(\delta):=[X \rightarrow Y \rightarrow Z]$, see [24, Section 3.3].

(5) All extension-closed subcategories of extriangulated categories are again extriangulated, see [24, Remark 2.18].

(6) Nakaoka and Palu in 24 provided a construction for which extriangulated categories are neither exact nor triangulated. That is, let $\mathcal{T}$ be an extriangulated category and $\mathcal{X}$ a full subcategory of $\mathcal{T}$. Denote by $\mathcal{P}$ (resp. $\mathcal{I}$ ) the full subcategory consisting of projective (resp. injective) objects in $\mathcal{T}$. If $\mathcal{X} \subseteq \mathcal{P} \cup \mathcal{I}$, then the quotient category $\mathcal{T} / \mathcal{X}$ is an extriangulated category, see [24, Proposition 3.30] for more details.

(7) Zhou and Zhu in [31, Corollary 4.12 and Remark 4.13] also provided a construction for which extriangulated categories are neither exact nor triangulated. That is, let $\mathcal{T}$ be a Krull-Schmidt triangulated category with Auslander-Reiten translation $\tau$ and $\mathcal{X}$ a functorially finite subcategory of $\mathcal{T}$ which satisfies $\tau \mathcal{X}=\mathcal{X}$. For any $X, Z \in \mathcal{T}$, define $\mathbb{E}(Z, X) \subseteq$ $\mathcal{T}(Z, X[1])$ to be the collection of equivalence classes of triangles $X \stackrel{f}{\rightarrow} Y \rightarrow Z \stackrel{\delta}{\rightarrow} X[1]$ 
such that $\mathcal{T}\left(f, X^{\prime}\right)$ is epic for any $X^{\prime} \in \mathcal{X}$, and define $\mathfrak{s}(\delta):=[X \rightarrow Y \rightarrow Z]$. Then $(\mathcal{T}, \mathbb{E}, \mathfrak{s})$ is a Frobenius extriangulated category. If $\mathcal{X} \neq\{0\}$, then $(\mathcal{T}, \mathbb{E}, \mathfrak{s})$ is not triangulated; and if $\mathcal{X} \neq \mathcal{T}$, then it is not exact.

Remark 2.9. Let $(\mathscr{C}, \mathbb{E}, \mathfrak{s})$ be a triple satisfying (ET1) and (ET2), and let $A \stackrel{x}{\longrightarrow} B \stackrel{y}{\longrightarrow} C \stackrel{\delta}{\rightarrow}$ be an $\mathbb{E}$-triangle.

(1) For any $a \in \mathscr{C}\left(A, A^{\prime}\right)$, there exists a morphism of $\mathbb{E}$-triangles

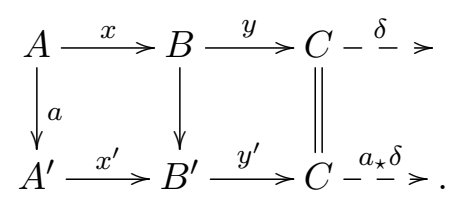

(2) For any $c \in \mathscr{C}\left(C^{\prime}, C\right)$, there exists a morphism of $\mathbb{E}$-triangles

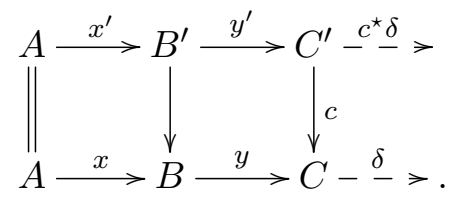

We introduce the following

Definition 2.10. Let $(\mathscr{C}, \mathbb{E}, \mathfrak{s})$ be a triple satisfying $(\mathrm{ET} 1)$ and $(\mathrm{ET} 2)$. An object $E \in \mathscr{C}$ is said to be injective if for any $\mathbb{E}$-triangle $A \stackrel{x}{\rightarrow} B \stackrel{y}{\rightarrow} C \stackrel{\delta}{\rightarrow}$ and each morphism $e \in$ $\mathscr{C}(A, E)$, there exists $b \in \mathscr{C}(B, E)$ such that $e=b x$.

We call an $\mathbb{E}$-triangle $A \stackrel{x}{\rightarrow} B \stackrel{y}{\rightarrow} C \stackrel{\delta}{>}$ split if the morphism $x$ is a section.

Lemma 2.11. Let $(\mathscr{C}, \mathbb{E}, \mathfrak{s})$ be a triple satisfying $(\mathrm{ET} 1)$, (ET2) and (ET3). Then the following statements are equivalent for an object $E \in \mathscr{C}$.

(1) E is injective.

(2) $\mathbb{E}(C, E)=0$ for any $C \in \mathscr{C}$.

(3) Any $\mathbb{E}$-triangle $E \rightarrow B \rightarrow C \stackrel{\delta}{\rightarrow}$ splits.

Proof. $(1) \Rightarrow(2)$. Let $\delta \in \mathbb{E}(C, E)$ and $\mathfrak{s}(\delta)=[E \stackrel{e}{\rightarrow} B \stackrel{y}{\rightarrow} C]$. Since $E$ is injective by (1), there exists $b \in \mathscr{C}(B, E)$ such that $b e=\mathrm{id}_{E}$; that is, we have the following commutative diagram

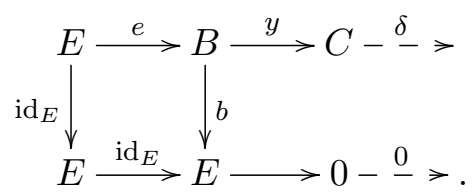


By (ET3), we get a morphism of $\mathbb{E}$-triangles

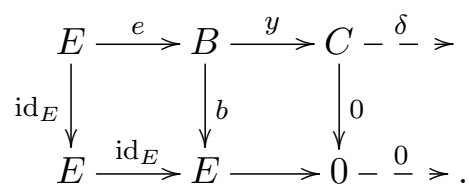

Thus we have $\delta=0^{\star} 0=0$.

$(2) \Rightarrow(3)$. It is trivial by [24, Remark 2.11].

$(3) \Rightarrow(1)$. Let $A \stackrel{x}{\rightarrow} B \stackrel{y}{\rightarrow} C \stackrel{\delta}{\rightarrow}$ be any $\mathbb{E}$-triangle. Then for any $a \in \mathscr{C}(A, E)$, there exists a morphism of $\mathbb{E}$-triangles

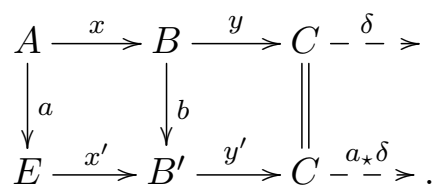

By assumption, the bottom $\mathbb{E}$-triangle splits, and hence there exists $b^{\prime} \in \mathscr{C}\left(B^{\prime}, E\right)$ such that $b^{\prime} x^{\prime}=\operatorname{id}_{E}$. Thus we have that $\left(b^{\prime} b\right) x=b^{\prime} x^{\prime} a=a$ and $E$ is injective.

Let $(\mathscr{C}, \mathbb{E}, \mathfrak{s})$ be a triple satisfying $(\mathrm{ET} 1)$ and $(\mathrm{ET} 2)$. We say that it has enough injective objects if for any $A \in \mathscr{C}$, there exists an $\mathbb{E}$-triangle $A \stackrel{x}{\rightarrow} E \stackrel{y}{\rightarrow} C \stackrel{\delta}{\rightarrow}$ with $E$ an injective object.

\section{Phantom morphisms}

\subsection{Phantom morphisms in additive categories}

Definition 3.1. Let $\mathbb{F}$ be an additive subfunctor of $\mathbb{E}$ and $\varphi \in \mathscr{C}(X, C)$. We call $\varphi$ an $\mathbb{F}$-phantom morphism if $\varphi^{\star} \delta \in \mathbb{F}(X, A)$ for any $\delta \in \mathbb{E}(C, A)$. Dually, let $\psi \in \mathscr{C}(A, Y)$. We call $\psi$ an $\mathbb{F}$-cophantom morphism if $\psi_{\star} \delta \in \mathbb{F}(C, Y)$ for any $\delta \in \mathbb{E}(C, A)$.

We denote by $\mathbf{P h}(\mathbb{F})$ and $\mathbf{C o p h}(\mathbb{F})$ the classes of $\mathbb{F}$-phantom and $\mathbb{F}$-cophantom morphisms respectively. In this paper, we only discuss the properties of $\mathbb{F}$-phantom morphisms in most cases, but we need to keep in mind that the dual results hold true for $\mathbb{F}$-cophantom morphisms, and we will directly use it if necessary.

We first note that $\mathbf{P h}(\mathbb{F})$ is an ideal. Indeed, let $\varphi \in \mathscr{C}(X, C)$ be an $\mathbb{F}$-phantom morphism. If $f \in \mathscr{C}\left(X^{\prime}, X\right)$, then for any $\delta \in \mathbb{E}(C, A)$, we have $(\varphi f)^{\star} \delta=f^{\star}\left(\varphi^{\star} \delta\right)$. Since $\varphi^{\star} \delta \in \mathbb{F}(X, A)$, we have $(\varphi f)^{\star} \delta \in \mathbb{F}\left(X^{\prime}, A\right)$, and hence $\varphi f$ is an $\mathbb{F}$-phantom morphism. Similarly, let $g \in \mathscr{C}\left(A, A^{\prime}\right)$, by the equality $(g \varphi)^{\star} \delta=\varphi^{\star}\left(g^{\star} \delta\right)$, we have $g \varphi$ is an $\mathbb{F}$-phantom morphism. Moreover, if $\varphi_{1}, \varphi_{2} \in \mathscr{C}(X, C)$ are $\mathbb{F}$-phantom morphisms, then by the equality $\left(\varphi_{1}+\varphi_{2}\right)^{\star} \delta=\varphi_{1}^{\star} \delta+\varphi_{2}{ }^{\star} \delta$, we have that $\varphi_{1}+\varphi_{2}$ is also an $\mathbb{F}$-phantom morphism. Therefore $\mathbf{P h}(\mathbb{F})$ is an ideal. 
Example 3.2. Let $R$ be a ring and $\mathbb{E}=\operatorname{Ext}_{R}^{1}(-,-)$.

(1) If $\mathbb{F}=\operatorname{PExt}_{R}^{1}(-,-)$ is as in Example 2.8(2), then the $\mathbb{F}$-phantom morphism is the phantom morphism in [15] and the pure phantom morphism in [10].

(2) If $\mathbb{F}=\operatorname{GExt}_{R}^{1}(-,-)$ is as in Example $2.8(2)$, then the $\mathbb{F}$-(co)phantom morphism is called the Gorenstein (co)phantom morphism. The following is a concrete example. Let $R=k Q / I$ with $k$ a field, where $Q$ is the quiver

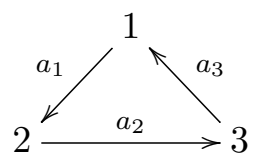

and $I=\left\langle a_{1} a_{3} a_{2}, a_{2} a_{1} a_{3}\right\rangle$. We can identify the irreducible Gorenstein cophantom morphisms in the category of finite generated left $R$-modules as follows:

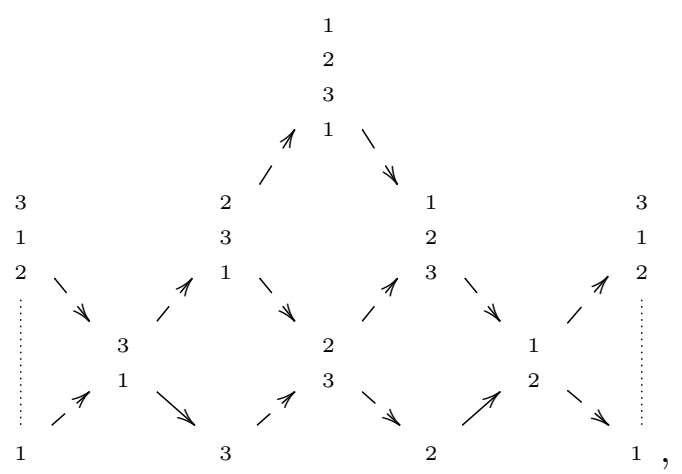

where the morphisms marked by the dashed arrows are all irreducible Gorenstein cophantom morphisms.

Let $\mathcal{I}$ be an ideal of $\mathscr{C}$. We write

$$
\begin{aligned}
& \mathcal{I}^{\star}:=\left\{i^{\star} \delta \mid i \in \mathcal{I} \text { and } \delta \text { is any } \mathbb{E} \text {-extension }\right\}, \\
& \mathcal{I}_{\star}:=\left\{i_{\star} \delta \mid i \in \mathcal{I} \text { and } \delta \text { is any } \mathbb{E} \text {-extension }\right\} .
\end{aligned}
$$

Proposition 3.3. $\mathcal{I}^{\star}$ is the minimum additive subfunctor of $\mathbb{E}$ for which $\mathcal{I} \subseteq \mathbf{P h}\left(\mathcal{I}^{\star}\right)$.

Proof. We first prove that $\mathcal{I}^{\star}$ is an additive subfunctor of $\mathbb{E}$. Let $\varphi \in \mathscr{C}(X, C)$ and $\delta \in \mathcal{I}^{\star}(C, A)$, that is, there exist $i \in \mathcal{I}\left(C, C^{\prime}\right)$ and $\delta^{\prime} \in \mathbb{E}\left(C^{\prime}, A\right)$ such that $\delta=i^{\star} \delta^{\prime}$. Then $\varphi^{\star} \delta=\varphi^{\star} i^{\star} \delta^{\prime}=(i \varphi)^{\star} \delta^{\prime}$. Since $\mathcal{I}$ is an ideal of $\mathscr{C}$, we have $i \varphi \in \mathcal{I}\left(X, C^{\prime}\right)$, and hence $\varphi^{\star} \delta \in \mathcal{I}^{\star}(X, A)$. Similarly, for $\psi \in \mathscr{C}(A, Y)$, by the equalities $\psi_{\star} \delta=\psi_{\star} i^{\star} \delta^{\prime}=i^{\star} \psi_{\star} \delta^{\prime}$, we have $\psi_{\star} \delta \in \mathcal{I}^{\star}(C, Y)$. The additivity of $\mathcal{I}^{\star}$ is induced by that of $\mathbb{E}$.

Next we prove the minimality about the property $\mathcal{I} \subseteq \mathbf{P h}\left(\mathcal{I}^{\star}\right)$. Let $\mathbb{F}$ be any additive subfunctor of $\mathbb{E}$ satisfying $\mathcal{I} \subseteq \mathbf{P h}(\mathbb{F})$. Let $\delta \in \mathcal{I}^{\star}(X, A)$, that is, there exist $i \in \mathcal{I}(X, C)$ and $\delta^{\prime} \in \mathbb{E}(C, A)$ such that $\delta=i^{\star} \delta^{\prime}$. Since $\mathcal{I} \subseteq \mathbf{P h}(\mathbb{F})$, we have $i \in \mathbf{P h}(\mathbb{F})$, and hence $\delta=i^{\star} \delta^{\prime} \in \mathbb{F}(X, A)$. This means that $\mathcal{I}^{\star} \subseteq \mathbb{F}$. 
Let $\mathcal{M}$ be a class of morphisms in $\mathscr{C}$. We denote by Mor $\mathscr{C}$ the collection of all morphisms in $\mathscr{C}$, and write

$$
\mathcal{M}^{\perp_{\mathbb{E}}}:=\left\{g \in \operatorname{Mor} \mathscr{C} \mid m^{\star} g_{\star} \delta=0 \text { for any } m \in \mathcal{M} \text { and any } \mathbb{E} \text {-extension } \delta\right\} .
$$

Then $\mathcal{M}^{\perp_{\mathbb{E}}}$ is an ideal of $\mathscr{C}$. Indeed, if $g_{1}, g_{2} \in \mathcal{M}^{\perp_{\mathbb{E}}}$, then for any $m \in \mathcal{M}$ and any $\mathbb{E}$-extension $\delta$, we have $m^{\star}\left(g_{1}+g_{2}\right)_{\star} \delta=m^{\star} g_{1_{\star}} \delta+m^{\star} g_{2{ }_{\star}} \delta=0$, which means that $g_{1}+g_{2} \in$ $\mathcal{M}^{\perp_{\mathbb{E}}}$. Let $g \in \mathcal{M}^{\perp_{\mathbb{E}}}$ and $h \in \operatorname{Mor} \mathscr{C}$ such that $h g$ is defined. Then $m^{\star}(h g)_{\star} \delta=m^{\star} h_{\star} g_{\star} \delta=$ $h_{\star} m^{\star} g_{\star} \delta=0$ implies that $h g \in \mathcal{M}^{\perp_{\mathbb{E}}}$. Similarly, if $g k$ is defined for $k \in$ Mor $\mathscr{C}$, then $g k \in \mathcal{M}^{\perp_{\mathbb{E}}}$. Therefore $\mathcal{M}^{\perp_{\mathbb{E}}}$ is an ideal of $\mathscr{C}$.

Dually, we write

$\perp_{\mathbb{E}} \mathcal{M}:=\left\{g \in \operatorname{Mor} \mathscr{C} \mid g^{\star} m_{\star} \delta=0\right.$ for any $m \in \mathcal{M}$ and any $\mathbb{E}$-extension $\left.\delta\right\}$.

Definition 3.4. (1) Let $f \in \mathscr{C}(X, C)$ and $g \in \mathscr{C}(A, Y)$. The pair $(f, g)$ is said to be $\mathbb{E}$-orthogonal if $f^{\star} g_{\star} \delta=0$ (or equivalently, $g_{\star} f^{\star} \delta=0$ ) for any $\delta \in \mathbb{E}(C, A)$.

(2) Let $\mathcal{I}$ and $\mathcal{J}$ be ideals of $\mathscr{C}$. The pair $(\mathcal{I}, \mathcal{J})$ is said to be $\mathbb{E}$-orthogonal if the pair $(i, j)$ is $\mathbb{E}$-orthogonal for any $i \in \mathcal{I}$ and $j \in \mathcal{J}$.

We write

$$
\mathbb{F} \text {-inj }:=\left\{i \in \operatorname{Mor} \mathscr{C} \mid i_{\star} \delta=0 \text { for each } \mathbb{F} \text {-extension } \delta\right\},
$$

and call the elements in $\mathbb{F}$-inj $\mathbb{F}$-injective morphisms. Dually, we write

$$
\mathbb{F} \text {-proj }:=\left\{i \in \operatorname{Mor} \mathscr{C} \mid i^{\star} \delta=0 \text { for each } \mathbb{F} \text {-extension } \delta\right\},
$$

and call the elements in $\mathbb{F}$-proj $\mathbb{F}$-projective morphisms.

Proposition 3.5. (1) The pair $(\mathbf{P h}(\mathbb{F}), \mathbb{F}$-inj) is $\mathbb{E}$-orthogonal.

(2) Let $\mathcal{I}$ be an ideal of $\mathscr{C}$. Then $\mathcal{I}^{\star}$-inj $=\mathcal{I}^{\perp_{\mathbb{E}}}$.

Proof. (1) It is clear.

(2) Let $j \in \mathcal{I}^{\star}$-inj. For any $i \in \mathcal{I}$ and any $\mathbb{E}$-extension $\delta$, we have that $i^{\star} \delta$ is an $\mathcal{I}^{\star}$-extension. So $i^{\star} j_{\star} \delta=j_{\star} i^{\star} \delta=0$ and $j \in \mathcal{I}^{\perp_{\mathbb{E}}}$. Conversely, let $j \in \mathcal{I}^{\perp_{\mathbb{E}}}$. For any $\mathcal{I}^{\star}$ extension $\delta$, there exist $i \in \mathcal{I}$ and an $\mathbb{E}$-extension $\delta^{\prime}$ such that $\delta=i^{\star} \delta^{\prime}$. So $j_{\star} \delta=j_{\star} i^{\star} \delta^{\prime}=$ $i^{\star} j_{\star} \delta^{\prime}=0$ and $j \in \mathcal{I}^{\star}$-inj.

\subsection{Phantom morphisms in extriangulated categories}

Let $(\mathscr{C}, \mathbb{E}, \mathfrak{s})$ be a triple satisfying $(\mathrm{ET} 1)$ and $(\mathrm{ET} 2)$, and let $A \stackrel{x}{\longrightarrow} B \stackrel{y}{\longrightarrow} C \stackrel{\delta}{\rightarrow}$ be any $\mathbb{E}$-triangle. Then for a morphism $\varphi \in \mathscr{C}(X, C)$, there exists a morphism of $\mathbb{E}$-triangles

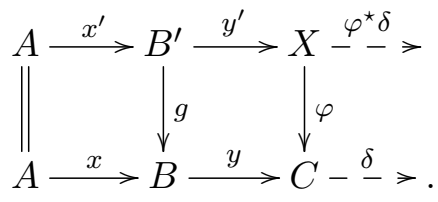


We easily see that $\varphi \in \mathscr{C}(X, C)$ is an $\mathbb{F}$-phantom morphism if and only if every $\mathbb{E}$-triangle $A \stackrel{x^{\prime}}{\rightarrow} B^{\prime} \stackrel{y^{\prime}}{\rightarrow} X^{\varphi^{\star} \delta} \rightarrow$ induced by the above is an $\mathbb{F}$-triangle.

Now let $(\mathscr{C}, \mathbb{E}, \mathfrak{s})$ be a triple satisfying $(\mathrm{ET} 1)$ and (ET2), and let $A \stackrel{x}{\rightarrow} B \stackrel{y}{\rightarrow} C \stackrel{\delta}{\rightarrow}$ be any $\mathbb{E}$-triangle and $\varphi \in \mathscr{C}(X, C)$ an $\mathbb{F}$-phantom morphism. For any $\mathbb{F}$-projective morphism $p \in \mathscr{C}(P, X)$, we have a morphism of $\mathbb{E}$-triangles

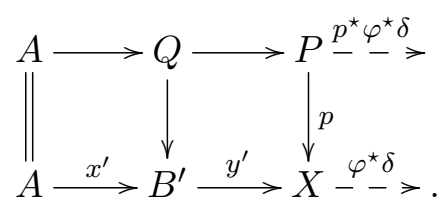

Since $\varphi^{\star} \delta$ is an $\mathbb{F}$-extension and $p$ is an $\mathbb{F}$-projective morphism, we have $p^{\star} \varphi^{\star} \delta=0$, and hence $(\varphi p)^{\star} \delta=0$, that is, the composition $P \stackrel{p}{\rightarrow} X \stackrel{\varphi}{\rightarrow} C$ is an $\mathbb{E}$-projective morphism.

Therefore, if we consider the stable category $(\mathscr{C}, \mathbb{E}, \mathfrak{s}):=(\mathscr{C}, \mathbb{E}, \mathfrak{s}) / \mathbb{E}$-proj, where the objects in $(\mathscr{C}, \mathbb{E}, \mathfrak{s})$ are the objects in $\mathscr{C}$, and for any $X, Y \in \mathscr{C}$, the morphism set $\operatorname{Hom}(X, Y)$ in $\overline{(\mathscr{C}, \mathbb{E}, \mathfrak{s})}$ are the morphism set $\mathscr{C}(X, Y) / \mathbb{E}$-proj, then $\mathbb{F}$-phantom morphisms make $\mathbb{F}$-projective morphisms vanish in $(\mathscr{C}, \mathbb{E}, \mathfrak{s})$. This is also why we call these morphisms "F-phantom" on some level.

Definition 3.6. Let $\mathcal{I}$ be an ideal of $\mathscr{C}$ and $C \in \mathscr{C}$.

(1) An $\mathcal{I}$-precover of $C$ is a morphism $i: X \rightarrow C$ in $\mathcal{I}$ such that any morphism $i^{\prime}: X^{\prime} \rightarrow C$ in $\mathcal{I}$ factors through $i$, that is, there exists a morphism $g: X^{\prime} \rightarrow X$ such that $i^{\prime}=i g$.

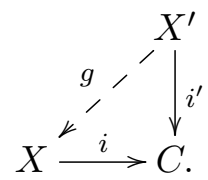

(2) Let $(\mathscr{C}, \mathbb{E}, \mathfrak{s})$ be a triple satisfying (ET1) and (ET2). A morphism $i: X \rightarrow C$ in $\mathcal{I}$ is called a special $\mathcal{I}$-precover of $C$ if there exists a morphism of $\mathbb{E}$-triangles

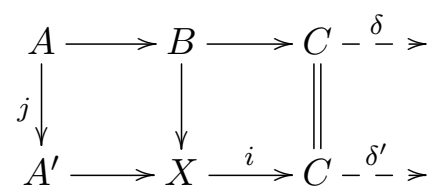

with $j \in \mathcal{I}^{\perp_{\mathbb{E}}}$.

An ideal $\mathcal{I}$ of $\mathscr{C}$ is called a (special) precovering ideal of $\mathscr{C}$ if any object in $\mathscr{C}$ admits an (a special) $\mathcal{I}$-precover. Dually, the notions of a (special) $\mathcal{I}$-preenvelope of an object and a (special) preenveloping ideal are defined.

In what follows, we always assume that the triple $(\mathscr{C}, \mathbb{E}, \mathfrak{s})$ satisfies $(\mathrm{ET} 1)$ and $(\mathrm{ET} 2)$. 
Proposition 3.7. Every special $\mathcal{I}$-precover is an $\mathcal{I}$-precover.

Proof. Let $C \in \mathscr{C}$, and $i: X \rightarrow C$ is a special $\mathcal{I}$-precover of $C$. Then there exists a morphism of $\mathbb{E}$-triangles

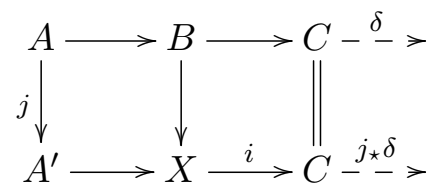

with $j \in \mathcal{I}^{\perp_{\mathbb{E}}}$. Now for any $i^{\prime}: X^{\prime} \rightarrow C$ in $\mathcal{I}$, there exists a morphism of $\mathbb{E}$-triangles

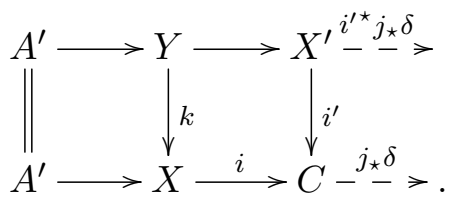

Since $i^{\prime} \in \mathcal{I}$ and $j \in \mathcal{I}^{\perp_{\mathbb{E}}}$, we have $i^{\prime \star} j_{\star} \delta=0$. So the morphism $Y \rightarrow X^{\prime}$ is a retraction and there exists $g: X^{\prime} \rightarrow Y$ such that $i^{\prime}=i k g$. It follows that $i: X \rightarrow C$ is an $\mathcal{I}$-precover of $C$.

Definition 3.8. An $\mathbb{E}$-orthogonal pair $(\mathcal{I}, \mathcal{J})$ of ideals of $\mathscr{C}$ is called an $\mathbb{E}$-cotorsion pair if $\mathcal{I}=\perp_{\mathbb{E}} \mathcal{J}$ and $\mathcal{J}=\mathcal{I}^{\perp_{\mathbb{E}}}$.

The following result gives a sufficient condition such that an $\mathbb{E}$-orthogonal pair of ideals is an $\mathbb{E}$-cotorsion pair.

Theorem 3.9. If $\mathcal{I}$ is a special precovering ideal, then the pair $\left(\mathcal{I}, \mathcal{I}^{\perp_{\mathbb{E}}}\right)$ of ideals is an $\mathbb{E}$-cotorsion pair.

Proof. Clearly, $\mathcal{I} \subseteq \perp_{\mathbb{E}}\left(\mathcal{I}^{\perp_{\mathbb{E}}}\right)$. Now let $i^{\prime} \in{ }^{\perp_{\mathbb{E}}}\left(\mathcal{I}^{\perp_{\mathbb{E}}}\right)$ with $i^{\prime}: X^{\prime} \rightarrow C$. For the object $C$, take a special $\mathcal{I}$-precover $i: X \rightarrow C$. Then there exists a morphism of $\mathbb{E}$-triangles

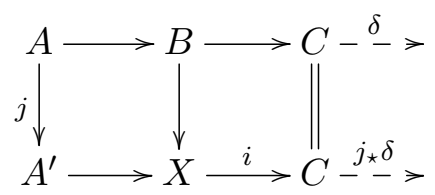

with $j \in \mathcal{I}^{\perp_{\mathbb{E}}}$. Furthermore, assume that $\mathfrak{s}\left(i^{\prime \star} j_{\star} \delta\right)=\left[A^{\prime} \stackrel{x}{\rightarrow} Y \stackrel{y}{\rightarrow} X^{\prime}\right]$. Then we also have a morphism of $\mathbb{E}$-triangles

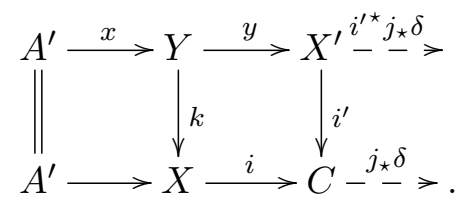

Since $i^{\prime} \in \perp_{\mathbb{E}}\left(\mathcal{I}^{\perp_{\mathbb{E}}}\right)$ and $j \in \mathcal{I}^{\perp_{\mathbb{E}}}$, we have $i^{\prime \star} j_{\star} \delta=0$, and hence there exists $y^{\prime}: X^{\prime} \rightarrow Y$ such that $i^{\prime}=i\left(k y^{\prime}\right)$. Thus we have that $i^{\prime} \in \mathcal{I}$ and ${ }^{\perp_{\mathbb{E}}}\left(\mathcal{I}^{\perp_{\mathbb{E}}}\right) \subseteq \mathcal{I}$. Therefore $\mathcal{I}={ }_{\mathbb{E}}\left(\mathcal{I}^{\perp_{\mathbb{E}}}\right)$ and $\left(\mathcal{I}, \mathcal{I}^{\perp_{\mathbb{E}}}\right)$ is an $\mathbb{E}$-cotorsion pair. 
Corollary 3.10. If $\mathcal{I}$ is a special precovering ideal, then $\mathcal{I}=\mathbf{P h}\left(\mathcal{I}^{\star}\right)$.

Proof. By definition, we have $\mathcal{I} \subseteq \mathbf{P h}\left(\mathcal{I}^{\star}\right)$. Now let $\varphi \in \mathbf{P h}\left(\mathcal{I}^{\star}\right)$, that is, $\varphi$ is an $\mathcal{I}^{\star}$ phantom morphism. Then for any $\mathbb{E}$-extension $\delta$, we have that $\varphi^{\star} \delta$ is an $\mathcal{I}^{\star}$-extension. Let $j \in \mathcal{I}^{\perp_{\mathbb{E}}}$. Since $\mathcal{I}^{\perp_{\mathbb{E}}}=\mathcal{I}^{\star}$-inj by Proposition $3.5(2)$, we have $j \in \mathcal{I}^{\star}$-inj. So $\varphi^{\star} j_{\star} \delta=$ $j_{\star} \varphi^{\star} \delta=0$ and $\varphi \in{ }^{\perp_{\mathbb{E}}}\left(\mathcal{I}^{\perp_{\mathbb{E}}}\right)$. Furthermore, $\mathcal{I}=\perp_{\mathbb{E}}\left(\mathcal{I}^{\perp_{\mathbb{E}}}\right)$ by Theorem 3.9. So $\varphi \in \mathcal{I}$ and $\operatorname{Ph}\left(\mathcal{I}^{\star}\right) \subseteq \mathcal{I}$

From Proposition $3.5(1)$, we have known that $(\mathbf{P h}(\mathbb{F}), \mathbb{F}$-inj) is an $\mathbb{E}$-orthogonal pair. In the rest of this section, we mainly study when it is an $\mathbb{E}$-cotorsion pair. To do it, we first introduce the following

Definition 3.11. (1) An additive subfunctor $\mathbb{F}$ of $\mathbb{E}$ is said to have enough injective morphisms if for any $A \in \mathscr{C}$, there exists an $\mathbb{F}$-triangle $A \stackrel{e}{\rightarrow} B \rightarrow C \stackrel{\delta}{\rightarrow}$, where $e$ is an $\mathbb{F}$-injective morphism.

(2) The additive subfunctor $\mathbb{F}$ of $\mathbb{E}$ is said to have enough special injective morphisms if for any $A \in \mathscr{C}$, there exists an $\mathbb{F}$-triangle as above together with a morphism of $\mathbb{E}$-triangles

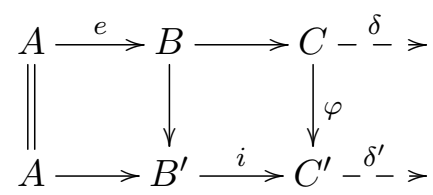

with $\varphi$ an $\mathbb{F}$-phantom morphism.

Lemma 3.12. Let $(\mathscr{C}, \mathbb{E}, \mathfrak{s})$ be a triple satisfying (ET1), (ET2) and (ET3). If an $\mathbb{F}$ inflation $x: A \rightarrow B$ factors through an $\mathbb{E}$-inflation $g: A \rightarrow Y$, then $g$ is an $\mathbb{F}$-inflation.

Proof. Since $x: A \rightarrow B$ is an $\mathbb{F}$-inflation, there exists an $\mathbb{F}$-triangle $A \stackrel{x}{\rightarrow} B \rightarrow C \stackrel{\delta}{\rightarrow}$; since $g: A \rightarrow Y$ is an $\mathbb{E}$-inflation, there exists an $\mathbb{E}$-triangle $A \stackrel{g}{\rightarrow} Y \rightarrow Z \stackrel{\delta^{\prime}}{\rightarrow}$ together with the following commutative diagram

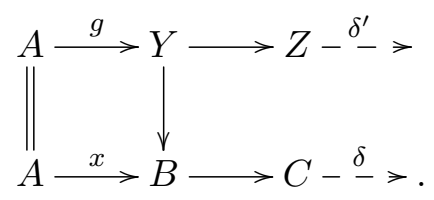

By (ET3), we get a morphism of $\mathbb{E}$-triangles

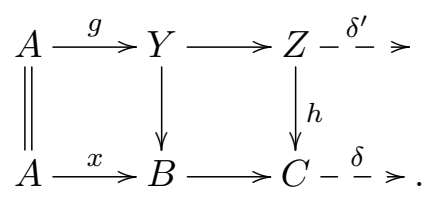

In particular, we have $\delta^{\prime}=h^{\star} \delta$. So $\delta^{\prime}$ is an $\mathbb{F}$-extension and $g$ is an $\mathbb{F}$-inflation. 
Proposition 3.13. Let $(\mathscr{C}, \mathbb{E}, \mathfrak{s})$ be a triple satisfying $(\mathrm{ET} 1),(\mathrm{ET} 2)$ and $(\mathrm{ET} 3)$. If $\mathbb{F} \subseteq \mathbb{E}$ is an additive subfunctor having enough injective morphisms, then $\mathbf{P h}(\mathbb{F})=\perp_{\mathbb{E}}(\mathbb{F}$-inj $)$.

Proof. By Proposition 3.5 (1), we have $\mathbf{P h}(\mathbb{F}) \subseteq \perp_{\mathbb{E}}(\mathbb{F}$-inj).

Now let $f: X \rightarrow C \in \perp_{\mathbb{E}}(\mathbb{F}$-inj $)$, and let $A \rightarrow B \rightarrow C \stackrel{\delta}{\rightarrow}$ be any $\mathbb{E}$-triangle. Then we have a morphism of $\mathbb{E}$-triangles

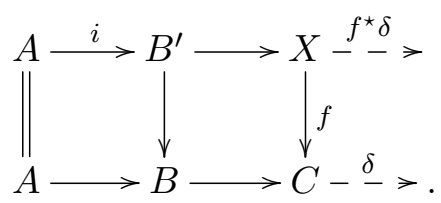

For the object $A$, by assumption there exists an $\mathbb{F}$-injective $\mathbb{F}$-inflation $e: A \rightarrow Y$. Consider the following morphism of $\mathbb{E}$-triangles

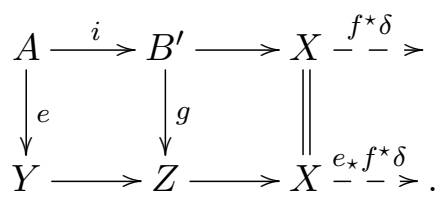

Since $e_{\star} f^{\star} \delta=f^{\star} e_{\star} \delta=0$, that is, the $\mathbb{E}$-triangle $Y \rightarrow Z \rightarrow X^{e_{\star}} \stackrel{f^{\star} \delta}{\rightarrow}$ splits, there exists $h: Z \rightarrow Y$ such that $e=(h g) i$. By Lemma 3.12, $i$ is also an $\mathbb{F}$-inflation. So each $A \stackrel{i}{\rightarrow} B^{\prime} \longrightarrow X \stackrel{f^{\star} \delta}{\rightarrow}$ induced by any $\mathbb{E}$-triangle along $f$ is an $\mathbb{F}$-triangle, which implies that $f$ is an $\mathbb{F}$-phantom morphism. Thus ${ }_{\mathbb{E}}(\mathbb{F}$-inj) $\subseteq \mathbf{P h}(\mathbb{F})$, and therefore $\mathbf{P h}(\mathbb{F})=$ $\perp_{\mathbb{E}}(\mathbb{F}$-inj).

Note that a morphism $e: A \rightarrow X$ in $\mathcal{I}$ is called a special $\mathcal{I}$-preenvelope of $A$ if there exists a morphism of $\mathbb{E}$-triangles

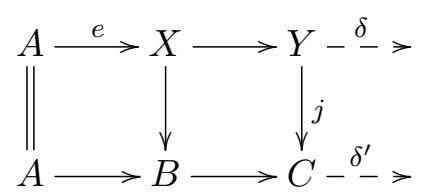

with $j \in{ }_{\mathbb{E}} \mathcal{I}$.

Now if $\mathbb{F}$ has enough special injective morphisms, then for any $A \in \mathscr{C}$, there exists an $\mathbb{F}$-triangle $A \stackrel{e}{\longrightarrow} X \rightarrow Y \stackrel{\delta}{\rightarrow}$ together with a morphism of $\mathbb{E}$-triangles

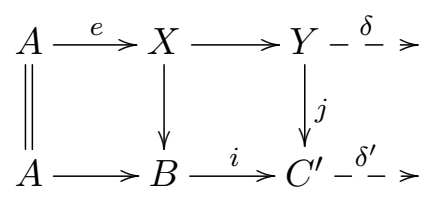


with $e \in \mathbb{F}$-inj and $j \in \mathbf{P h}(\mathbb{F})$. By Proposition 3.13, we have $\mathbf{P h}(\mathbb{F})=\perp_{\mathbb{E}}(\mathbb{F}$-inj $)$. So $j \in \perp_{\mathbb{E}}(\mathbb{F}$-inj) and $e$ is a special $\mathbb{F}$-injective preenvelope of $A$. This shows that $\mathbb{F}$-inj is a special preenveloping ideal.

As a dual of Theorem 3.9, we have the following

Theorem 3.14. If $\mathcal{J}$ is a special preenveloping ideal of $\mathscr{C}$, then the orthogonal pair $\left(\perp_{\mathbb{E}} \mathcal{J}, \mathcal{J}\right)$ of ideals is an $\mathbb{E}$-cotorsion pair.

Note that if $\mathbb{F}$ has enough special injective morphisms, then $\left(\perp_{\mathbb{E}}(\mathbb{F}\right.$-inj $), \mathbb{F}$-inj $)$ is an $\mathbb{E}$ cotorsion pair of ideals by Theorem 3.14. Because $\mathbf{P h}(\mathbb{F})=\perp_{\mathbb{E}}(\mathbb{F}$-inj) by Proposition 3.13 . we get the following

Corollary 3.15. Let $(\mathscr{C}, \mathbb{E}, \mathfrak{s})$ be a triple satisfying (ET1), (ET2) and (ET3). If $\mathbb{F}$ has enough special injective morphisms, then $(\mathbf{P h}(\mathbb{F}), \mathbb{F}$-inj) is an $\mathbb{E}$-cotorsion pair of ideals; in particular, $\mathbf{P h}(\mathbb{F})^{\perp_{\mathbb{E}}}=\mathbb{F}$-inj.

4. The interplay between phantom ideals and cotorsion pairs

From the previous section, we know that a special precovering ideal corresponds an $\mathbb{E}$ cotorsion pair, and that a phantom ideal induced by a subfunctor also corresponds ones under a suitable assumption. In this section, we will investigate their interplay by showing that a phantom ideal induced by a subfunctor is a special precovering ideal under some suitable assumption, and vice versa. Before doing it, we first give the following lemma, which simplifies the calculation process for checking a morphism to be phantom.

Lemma 4.1. Let $(\mathscr{C}, \mathbb{E}, \mathfrak{s})$ be an extriangulated category. Consider an $\mathbb{E}$-triangle $K \rightarrow P \stackrel{p}{\rightarrow} C \stackrel{\gamma}{\rightarrow}$ with $p$ an $\mathbb{E}$-projective morphism and a morphism $\varphi: X \rightarrow C$. Then the following statements are equivalent.

(1) $\varphi$ is an $\mathbb{F}$-phantom morphism.

(2) The induced $\mathbb{E}$-triangle $K \rightarrow Y \rightarrow X_{-}^{\varphi^{\star} \gamma} \underset{>}{>}$ is an $\mathbb{F}$-triangle.

Proof. $(1) \Rightarrow(2)$. It is trivial.

$(2) \Rightarrow(1)$. Let $A \rightarrow B \stackrel{y}{\rightarrow} C \stackrel{\delta}{\rightarrow}$ be any $\mathbb{E}$-triangle. By 24 , Proposition 3.15], we have the following commutative diagram 


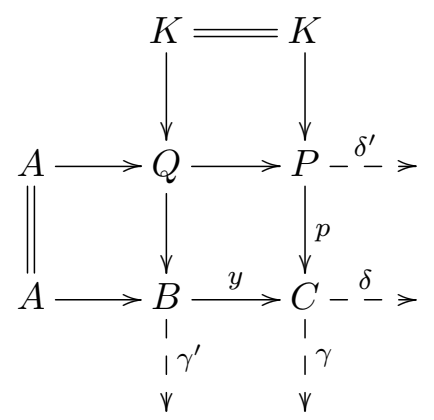

in $\mathscr{C}$ with $\delta^{\prime}=p^{\star} \delta$ and $\gamma^{\prime}=y^{\star} \gamma$. Since $p$ is an $\mathbb{E}$-projective morphism, we have $\delta^{\prime}=p^{\star} \delta=$ 0 , and hence the middle row splits. Then there exists $g: P \rightarrow B$ such that $p=y g$, that is, the following diagram

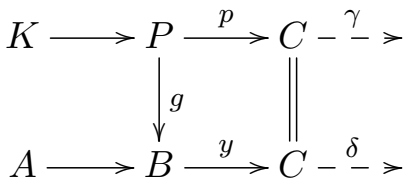

is commutative. By $(\mathrm{ET} 3)^{\mathrm{op}}$, there exists a morphism of $\mathbb{E}$-triangles

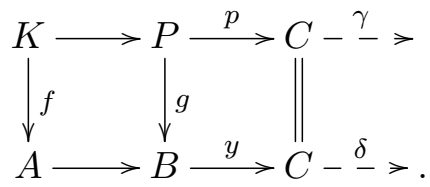

In particular, we have $\delta=f_{\star} \gamma$. Thus $\varphi^{\star} \delta=\varphi^{\star} f_{\star} \gamma=f_{\star} \varphi^{\star} \gamma$. By assumption, $\varphi^{\star} \gamma$ is an $\mathbb{F}$-extension, and hence $\varphi^{\star} \delta$ is also an $\mathbb{F}$-extension, which shows that $\varphi: X \rightarrow C$ is an $\mathbb{F}$-phantom morphism.

Now we show that, under a suitable assumption, phantom ideals induced by additive subfunctors having enough injective morphisms are special precovering ideals.

Theorem 4.2. Let $(\mathscr{C}, \mathbb{E}, \mathfrak{s})$ be an extriangulated category with enough projective morphisms, and assume that $\mathbb{F} \subseteq \mathbb{E}$ is an additive subfunctor having enough injective morphisms. Then $\mathbf{P h}(\mathbb{F})$ is a special precovering ideal.

Proof. Let $C \in \mathscr{C}$. Then by assumption, there exists an $\mathbb{E}$-triangle $K \rightarrow P \stackrel{p}{\rightarrow} C \stackrel{\gamma}{\rightarrow}$ with $p$ an $\mathbb{E}$-projective morphism. For the object $K$, there exists an $\mathbb{F}$-injective $\mathbb{F}$-inflation $e: K \rightarrow X$. Then we get a morphism of $\mathbb{E}$-triangles

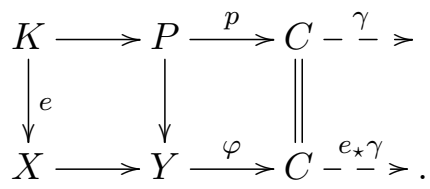


In the following, we argue that $\varphi$ is a special $\mathbf{P h}(\mathbb{F})$-precover of $C$.

First of all, by Proposition $3.5(1)$, we have that $\mathbb{F}$-inj $\subseteq \mathbf{P h}(\mathbb{F})^{\perp_{\mathbb{E}}}$ and $e \in \mathbf{P h}(\mathbb{F})^{\perp_{\mathbb{E}}}$. Moreover, consider the following diagram of morphisms of $\mathbb{E}$-triangles

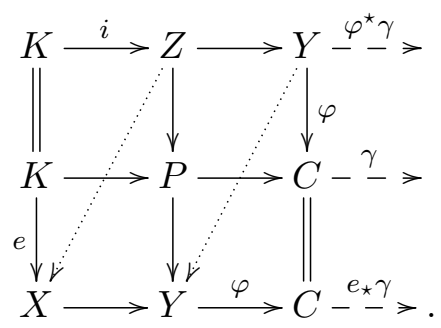

Since $\operatorname{id}_{C} \varphi=\varphi=\varphi \operatorname{id}_{Y}$, there exists $z: Z \rightarrow X$ such that $e=e \operatorname{id}_{K}=z i$ by 24 , Corollary 3.5]. Since $e$ is an $\mathbb{F}$-inflation, $i$ is also an $\mathbb{F}$-inflation by Lemma 3.12, and hence $\varphi^{\star} \gamma$ is an $\mathbb{F}$-extension. By Lemma 4.1, $\varphi$ is an $\mathbb{F}$-phantom morphism and it is a special $\mathbf{P h}(\mathbb{F})$-precover of $C$.

Therefore we conclude that $\mathbf{P h}(\mathbb{F})$ is a special precovering ideal.

Lemma 4.3. Let $\mathcal{M}$ be a class of morphisms in $\mathscr{C}$. Consider a morphism of $\mathbb{E}$-triangles

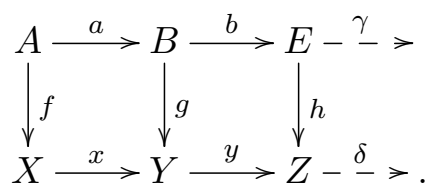

If $f \in \mathcal{M}^{\perp_{\mathbb{E}}}$ and $E$ is an injective object, then $g \in \mathcal{M}^{\perp_{\mathbb{E}}}$.

Proof. Since $\mathcal{M}^{\perp_{\mathbb{E}}}$ is an ideal and $f \in \mathcal{M}^{\perp_{\mathbb{E}}}$, we have $g a=x f \in \mathcal{M}^{\perp_{\mathbb{E}}}$. Thus for any $m \in \mathcal{M}$, we have $m^{\star} g_{\star} a_{\star}=m^{\star}(g a)_{\star}=0$. On one hand, by Lemma 2.11 we have that an object $E \in \mathscr{C}$ is injective if and only if $\mathbb{E}(C, E)=0$ for any $C \in \mathscr{C}$. On the other hand, by [24. Corollary 3.12], there exists an exact sequence

$$
\mathbb{E}(C, A) \stackrel{a_{\star}}{\longrightarrow} \mathbb{E}(C, B) \rightarrow \mathbb{E}(C, E) .
$$

Thus $a_{\star}$ is epic and $m^{\star} g_{\star}=0$, which shows that $g \in \mathcal{M}^{\perp_{\mathbb{E}}}$.

Theorem 4.4. Let $(\mathscr{C}, \mathbb{E}, \mathfrak{s})$ be an extriangulated category with enough injective objects. If $\mathcal{I}$ is a special precovering ideal, then $\mathcal{I}^{\perp_{\mathbb{E}}}$ is a special preenveloping ideal.

Proof. Let $A \in \mathscr{C}$ and $A \longrightarrow E \stackrel{c}{\longrightarrow} C \stackrel{\delta}{\rightarrow}$ be an $\mathbb{E}$-triangle with $E$ an injective object. For the object $C$, there exists a special $\mathcal{I}$-precover $x: X \rightarrow C$. Then we have a morphism of $\mathbb{E}$-triangles

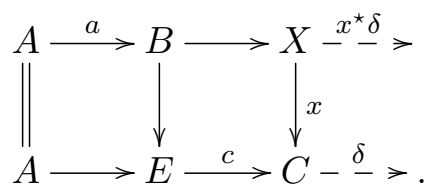


In the following, we argue that $a$ is a special $\mathcal{I}^{\perp_{\mathbb{E}}}$-preenvelope of $A$.

Since $x \in \mathcal{I} \subseteq \perp_{\mathbb{E}}\left(\mathcal{I}^{\perp_{\mathbb{E}}}\right)$, it suffices to show that $a \in \mathcal{I}^{\perp_{\mathbb{E}}}$. Assume that the special $\mathcal{I}$-precover $x: X \rightarrow C$ comes from the following morphism of $\mathbb{E}$-triangles

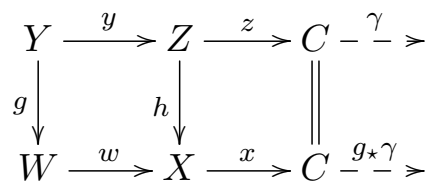

with $g \in \mathcal{I}^{\perp_{\mathbb{E}}}$. Consider the following commutative diagram

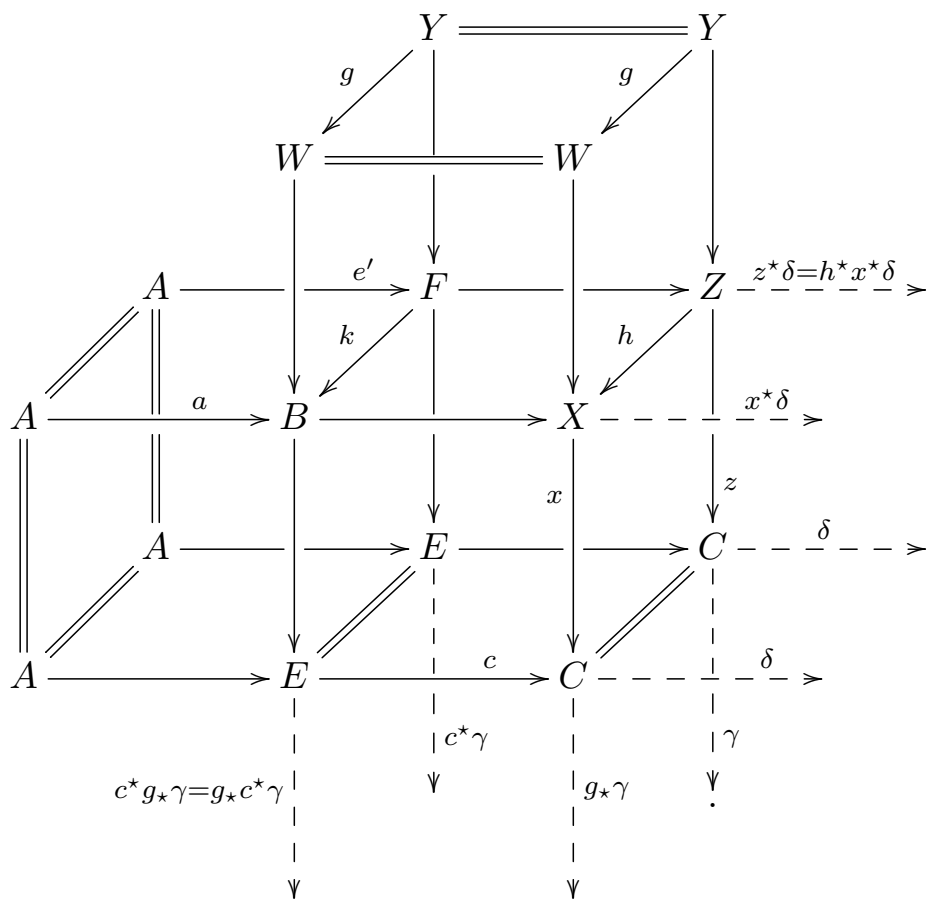

By Lemma 4.3 and the vertical plane in the middle of the above diagram, we have $k \in \mathcal{I}^{\perp_{\mathbb{E}}}$, and hence $a=k e^{\prime} \in \mathcal{I}^{\perp_{\mathbb{E}}}$, as desired.

Following the above theorem and its dual, we get a morphism version of the Salce's lemma as follows.

Lemma 4.5 (Salce's Lemma). Let $(\mathscr{C}, \mathbb{E}, \mathfrak{s})$ be an extriangulated category with enough projective and injective objects. If $(\mathcal{I}, \mathcal{J})$ is an $\mathbb{E}$-cotorsion pair of ideals, then $\mathcal{I}$ is a special precovering ideal if and only if $\mathcal{J}$ is a special preenveloping ideal.

Now we give our main result as follows, which extends [10, Theorem 1] from exact categories to extriangulated categories. Here, an $\mathbb{E}$-cotorsion pair $(\mathcal{I}, \mathcal{J})$ of ideals is called complete if $\mathcal{I}$ is a special precovering ideal and $\mathcal{J}$ is a special preenveloping ideal. 
Theorem 4.6. Let $(\mathscr{C}, \mathbb{E}, \mathfrak{s})$ be an extriangulated category. Then we have the following implications.

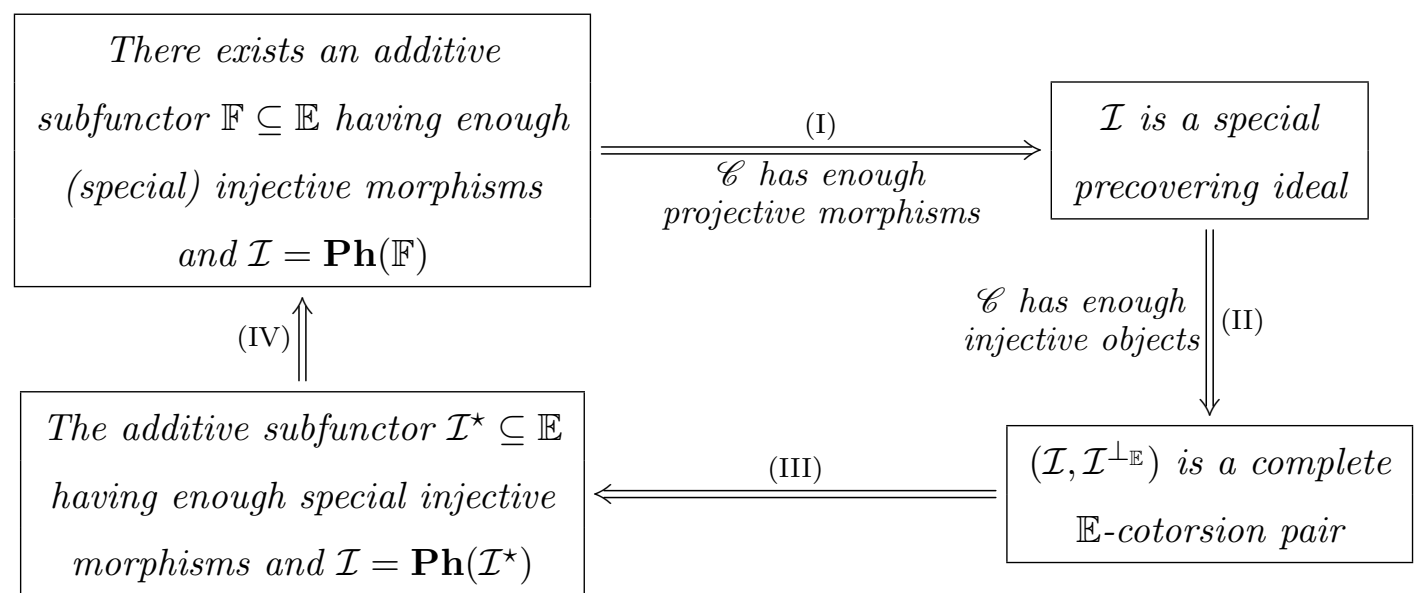

Proof. (I) It follows directly from Theorem 4.2 .

(II) Since $\mathcal{I}$ is a special precovering ideal, $\left(\mathcal{I}, \mathcal{I}^{\perp_{\mathbb{E}}}\right)$ is an $\mathbb{E}$-cotorsion pair by Theorem 3.9. Moreover, since $\mathscr{C}$ has enough injective objects, $\mathcal{I}^{\perp_{\mathbb{E}}}$ is a special preenveloping ideal by Theorem 4.4 . Thus $\left(\mathcal{I}, \mathcal{I}^{\perp_{\mathbb{E}}}\right)$ is a complete $\mathbb{E}$-cotorsion pair.

(III) First, since $\mathcal{I}$ is a special precovering ideal, we have $\mathcal{I}=\operatorname{Ph}\left(\mathcal{I}^{\star}\right)$ by Corollary 3.10 . Moreover, we have $\mathcal{I}^{\perp_{\mathbb{E}}}=\mathcal{I}^{\star}$-inj by Proposition 3.5(2). So by assumption, any object in $\mathscr{C}$ admits a special $\mathcal{I}^{\star}$-injective preenvelope, that is, for any $A \in \mathscr{C}$, there exists an $\mathcal{I}^{\star}$ injective morphism $e: A \rightarrow X$ that comes from a morphism of $\mathbb{E}$-triangles

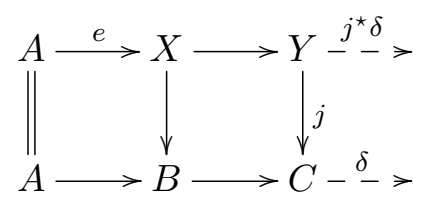

with $j \in \perp_{\mathbb{E}}\left(\mathcal{I}^{\star}\right.$-inj). This, on the other hand, shows that $\mathcal{I}^{\star}$ has enough injective morphisms. So by Proposition 3.13, we have that ${ }^{\perp_{\mathbb{E}}}\left(\mathcal{I}^{\star}\right.$-inj $)=\mathbf{P h}\left(\mathcal{I}^{\star}\right)$ and $j \in \mathbf{P h}\left(\mathcal{I}^{\star}\right)$. It follows that $\mathcal{I}^{\star}$ has enough special injective morphisms.

(IV) It is trivial.

By Theorem 4.6, we have that if $(\mathscr{C}, \mathbb{E}, \mathfrak{s})$ is an extriangulated category with enough injective objects and projective morphisms, then we get the following bijective correspondence.

$$
\begin{array}{|c|c|c|}
\hline \begin{array}{c}
\text { all special precovering ideals } \\
\text { of } \mathscr{C}
\end{array} & \stackrel{(-)^{\star}}{\underset{\mathbf{P h}(-)}{<}} \begin{array}{l}
\text { all additive subfunctors of } \mathbb{E} \text { having } \\
\text { enough special injective morphisms }
\end{array} \\
\hline
\end{array}
$$

Combining it with the Salce's lemma, we further get the following 
Theorem 4.7. Let $(\mathscr{C}, \mathbb{E}, \mathfrak{s})$ be an extriangulated category with enough injective objects and projective objects. Then we have the following implications for an $\mathbb{E}$-cotorsion pair $(\mathcal{I}, \mathcal{J})$ of ideals.

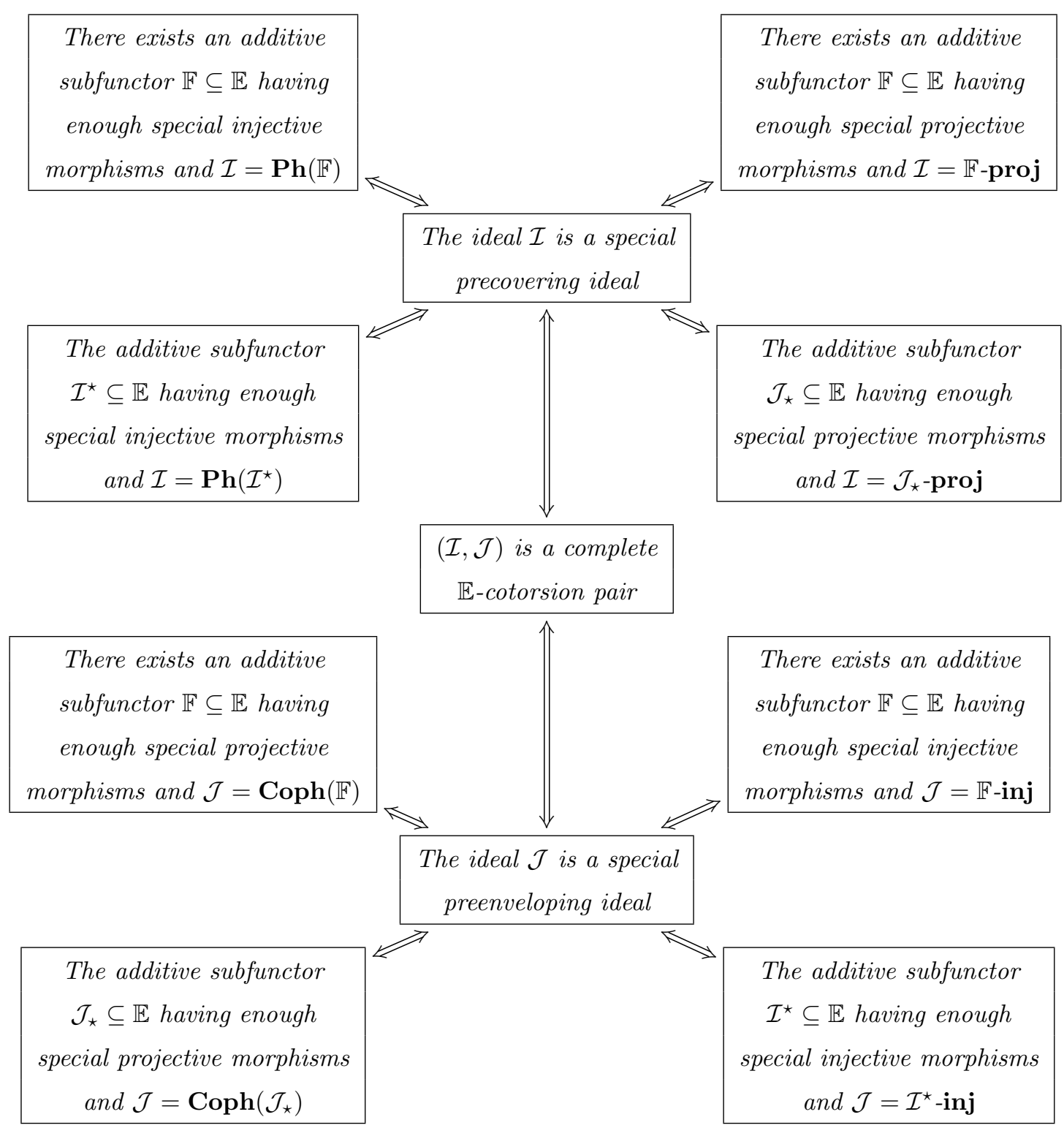

The above theorem shows that if $(\mathscr{C}, \mathbb{E}, \mathfrak{s})$ is an extriangulated category with enough injective objects and projective objects, then we have the following bijective correspondences. 
$(4.2)$

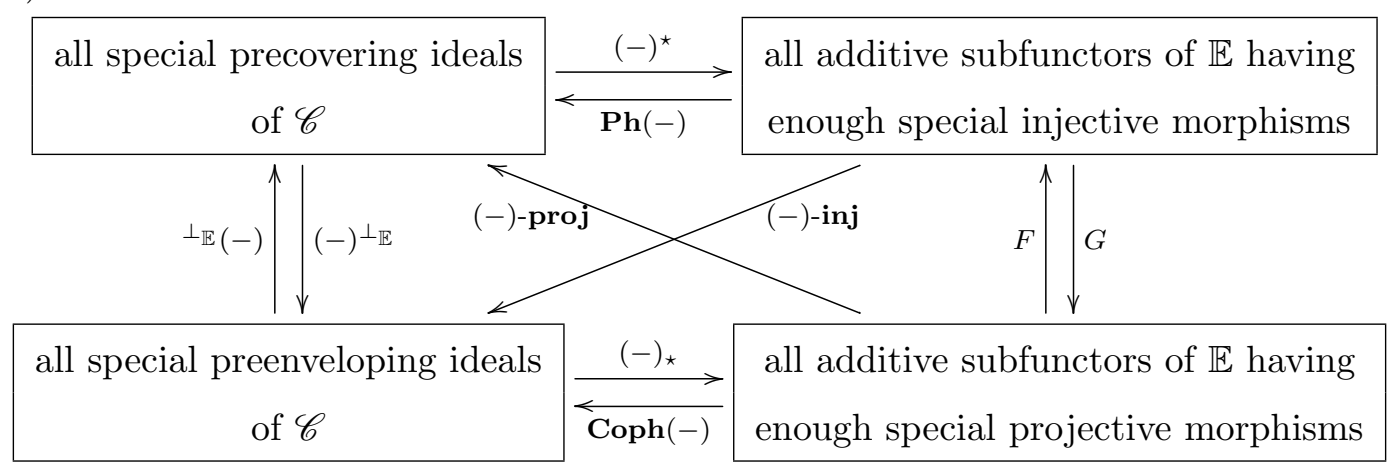

Here $F=(-)^{\star} \circ \perp_{\mathbb{E}}(-) \circ \mathbf{C o p h}(-)$ and $G=(-)_{\star} \circ(-)^{\perp_{\mathbb{E}}} \circ \mathbf{P h}(-)$.

We end this section with some applications of the obtained results above.

Theorem 4.8. Let $(\mathscr{C}, \mathbb{E}, \mathfrak{s})$ be an extriangulated category with enough injective objects and projective morphisms. If an additive subfunctor $\mathbb{F} \subseteq \mathbb{E}$ has enough injective morphisms, then we have

(1) The pair $\left(\perp_{\mathbb{E}}\left(\mathbb{F}\right.\right.$-inj), $\left.\left({ }_{\mathbb{E}}(\mathbb{F} \text {-inj })\right)^{\perp_{\mathbb{E}}}\right)$ of ideals generated by $\mathbb{F}$-inj is a complete $\mathbb{E}$ cotorsion pair of ideals.

(2) $\mathbf{P h}(\mathbb{F})^{\perp_{\mathbb{E}}}=\mathbf{P h}(\mathbb{F})^{\star}$-inj, and $\mathbf{P h}(\mathbb{F})^{\perp_{\mathbb{E}}}$ is the minimum ideal containing $\mathbb{F}$-inj and satisfying the following property $(\mathrm{C})$ : Let $\mathcal{I}$ be an ideal and consider a morphism of $\mathbb{E}$-triangles

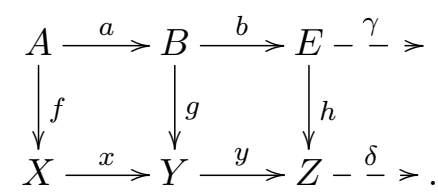

If $f \in \mathcal{I}$ and $E$ is an injective object, then $g \in \mathcal{I}$.

(3) The additive subfunctor $\mathbf{P h}(\mathbb{F})^{\star} \subseteq \mathbb{E}$ is the maximum additive subfunctor of $\mathbb{F}$ having enough special injective morphisms.

Proof. (1) By Proposition 3.13, we have $\perp_{\mathbb{E}}(\mathbb{F}$-inj $)=\mathbf{P h}(\mathbb{F})$. By Theorem 4.2, $\mathbf{P h}(\mathbb{F})$ is a special precovering ideal. Moreover, by Theorem $4.4 \quad \mathbf{P h}(\mathbb{F})^{\perp_{\mathbb{E}}}=\left(\perp_{\mathbb{E}}(\mathbb{F} \text {-inj } \mathbf{j})\right)^{\perp_{\mathbb{E}}}$ is a special preenveloping ideal. Thus $\left(\perp_{\mathbb{E}}(\mathbb{F}\right.$-inj $\left.),\left(\perp_{\mathbb{E}}(\mathbb{F} \text {-inj })\right)^{\perp_{\mathbb{E}}}\right)$ is a complete $\mathbb{E}$-cotorsion pair of ideals.

(2) By Proposition 3.5, $\mathbb{F}$-inj $\subseteq \mathbf{P h}(\mathbb{F})^{\perp_{\mathbb{E}}}=\mathbf{P h}(\mathbb{F})^{\star}$-inj. By Lemma 4.3, $\mathbf{P h}(\mathbb{F})^{\perp_{\mathbb{E}}}$ satisfies the property $(\mathrm{C})$. Now let $\mathcal{J}$ be an ideal of $\mathscr{C}$ containing $\mathbb{F}$-inj and satisfying the property $(\mathrm{C})$. We will show that $\mathbf{P h}(\mathbb{F})^{\perp_{\mathbb{E}}} \subseteq \mathcal{J}$. To do it, let $j \in \mathbf{P h}(\mathbb{F})^{\perp_{\mathbb{E}}}$ with $j: A \rightarrow J$. Consider the same commutative diagram as in the proof of Theorem 4.4. Since $\mathbb{F} \subseteq \mathbb{E}$ has 
enough injective morphisms, we can adjust the morphism $g: Y \rightarrow W$ to be in $\mathbb{F}$-inj. By the property $(\mathrm{C})$, we have that $k \in \mathcal{J}$ and $a=k e^{\prime} \in \mathcal{J}$. Moreover, by Theorem 4.4, the

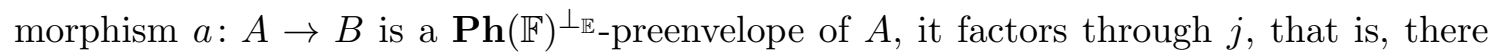
exists $b: B \rightarrow J$ such that $j=b a$, and thus $j \in \mathcal{J}$, as desired.

(3) Clearly, $\mathbf{P h}(\mathbb{F})^{\star} \subseteq \mathbb{F}$. Now since $\mathbf{P h}(\mathbb{F})$ is a special precovering ideal by Theorem $4.2, \mathbf{P h}(\mathbb{F})^{\star}$ is an additive subfunctor having enough special injective morphisms by the correspondence 4.1. Suppose that $\mathbb{F}^{\prime} \subseteq \mathbb{F}$ is an additive subfunctor having enough special injective morphisms. To show $\mathbb{F}^{\prime} \subseteq \mathbf{P h}(\mathbb{F})^{\star}$, it suffices to show that every $\mathbb{F}^{\prime}-$ triangle $A \longrightarrow B \longrightarrow C \stackrel{\delta}{\rightarrow}$ is a $\mathbf{P h}(\mathbb{F})^{\star}$-triangle.

Let $e: A \rightarrow X$ be a special $\mathbb{F}^{\prime}$-injective $\mathbb{F}^{\prime}$-inflation. Then we have a morphism of $\mathbb{F}^{\prime}$-triangles

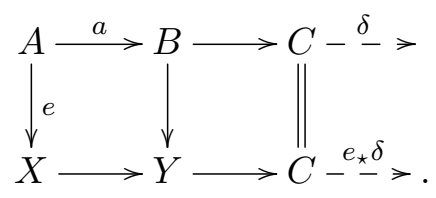

Since $e$ is $\mathbb{F}^{\prime}$-injective, we have that $e_{\star} \delta=0$ and there exists $b: B \rightarrow X$ such that $e=b a$. This also induces the following commutative diagram

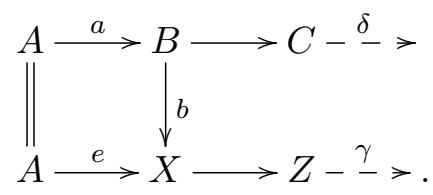

By (ET3), we get a morphism of $\mathbb{F}^{\prime}$-triangles

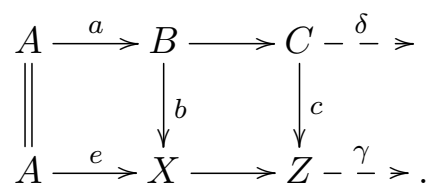

On the other hand, since $e: A \rightarrow X$ is a special $\mathbb{F}^{\prime}$-injective $\mathbb{F}^{\prime}$-inflation, by definition there exists a morphism of $\mathbb{E}$-triangles

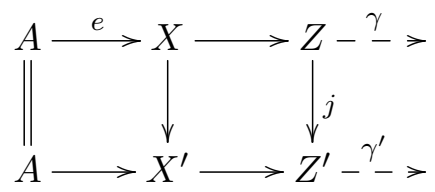

with $j \in \mathbf{P h}\left(\mathbb{F}^{\prime}\right) \subseteq \mathbf{P h}(\mathbb{F})$. Thus we get a morphism of $\mathbb{E}$-triangles

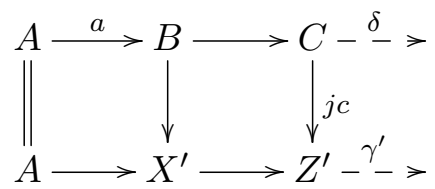

with $j c \in \mathbf{P h}(\mathbb{F})$. This shows that $\delta=(j c)^{\star} \gamma^{\prime} \in \mathbf{P h}(\mathbb{F})^{\star}$, as desired. 
Corollary 4.9. Let $(\mathscr{C}, \mathbb{E}, \mathfrak{s})$ be an extriangulated category with enough injective objects and projective morphisms. If $\mathbb{F} \subseteq \mathbb{E}$ is an additive subfunctor having enough injective morphisms, then the following statement are equivalent.

(1) The subfunctor $\mathbb{F}$ has enough special injective morphisms.

(2) $\mathbb{F}=\mathbf{P h}(\mathbb{F})^{\star}$.

(3) $\mathbf{P h}(\mathbb{F})^{\perp_{\mathbb{E}}}=\mathbb{F}$-inj.

Proof. (1) $\Rightarrow(2)$. It follows from Theorem 4.8(3).

$(2) \Rightarrow(3)$. By Proposition $3.5(2)$.

$(3) \Rightarrow(1)$. By Theorem $4.2, \mathbf{P h}(\mathbb{F})$ is a special precovering ideal. By Theorem 4.6(II), $\left(\mathbf{P h}(\mathbb{F}), \mathbf{P h}(\mathbb{F})^{\perp_{\mathbb{E}}}\right)=(\mathbf{P h}(\mathbb{F}), \mathbb{F}$-inj) is a complete $\mathbb{E}$-cotorsion pair, and hence $\mathbb{F}$-inj is a special preenveloping ideal, that is, for any $A \in \mathscr{C}$, there exists a morphism of $\mathbb{E}$-triangles

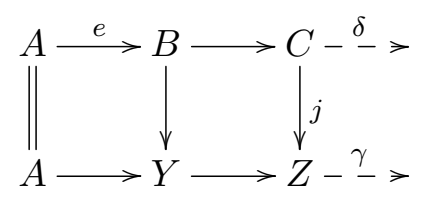

with $e \in \mathbb{F}$-inj and $j \in \perp_{\mathbb{E}}(\mathbb{F}$-inj). Moreover, since $(\mathbf{P h}(\mathbb{F}), \mathbb{F}$-inj) is an $\mathbb{E}$-cotorsion pair, we have that $\perp_{\mathbb{E}}(\mathbb{F}$-inj) $=\mathbf{P h}(\mathbb{F})$ and $j \in \mathbf{P h}(\mathbb{F})$. Thus $\mathbb{F}$ has enough special injective morphisms, as desired.

\section{The correspondences for object ideals}

From now on, objects are sometimes identified with the identity morphisms. Let $\mathcal{I}$ be a class of morphisms in $\mathscr{C}$. We write $\operatorname{Ob}(\mathcal{I}):=\left\{A \in \mathscr{C} \mid \operatorname{id}_{A} \in \mathcal{I}\right\}$, and denote by $\langle\mathcal{I}\rangle$ the smallest ideal of $\mathscr{C}$ containing $\mathcal{I}$. If $\mathcal{I}=\langle\operatorname{Ob}(\mathcal{I})\rangle$, then we call $\mathcal{I}$ an object ideal, that is, it is generated by itself objects. An object $A \in \mathscr{C}$ is called $\mathbb{F}$-injective if id $_{A} \in \mathbb{F}$-inj. It is easy to check that an object $A \in \mathscr{C}$ is $\mathbb{F}$-injective if and only if it is injective with respect to all $\mathbb{F}$-triangles.

Let $(\mathscr{C}, \mathbb{E}, \mathfrak{s})$ be an extriangulated category with enough projective morphisms and $\mathbb{F} \subseteq \mathbb{E}$ an additive subfunctor having enough injective objects. Then for any $C \in \mathscr{C}$, there is an $\mathbb{E}$-triangle $K \longrightarrow P \stackrel{p}{\rightarrow} C \stackrel{\gamma}{\rightarrow}$, with $p$ an $\mathbb{E}$-projective morphism. For the object $K$, by assumption there exists an $\mathbb{F}$-inflation $e: K \rightarrow E$ with $E$ an $\mathbb{F}$-injective object. Then we get a morphism of $\mathbb{E}$-triangles

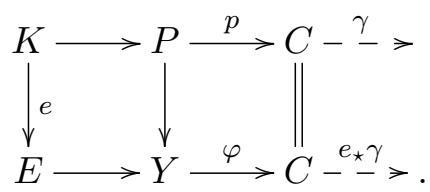


Since $E$ is an $\mathbb{F}$-injective object, that is, $\operatorname{id}_{E} \in \mathbb{F}$-inj, we have $e=\operatorname{id}_{E} e \in \mathbb{F}$-inj. Thus as in the proof of Theorem 4.2, the morphism $\varphi$ is an $\mathbb{F}$-phantom morphism. Therefore, for any $C \in \mathscr{C}$, there always exists an $\mathbb{E}$-triangle $E \rightarrow Y \stackrel{\varphi}{\rightarrow} C \stackrel{\delta}{\rightarrow}$ with $\varphi$ an $\mathbb{F}$-phantom morphism and $E$ an $\mathbb{F}$-injective object. Moreover, since $\mathbb{F}$-inj $\subseteq \mathbf{P h}(\mathbb{F})^{\perp_{\mathbb{E}}}$, the object $E$ is also in $\mathbf{P h}(\mathbb{F})^{\perp_{\mathbb{E}}}$. This allows us to give the following definition.

Definition 5.1. Let $\mathcal{I}$ be an ideal of $\mathscr{C}$. We call a morphism $i: X \rightarrow C$ in $\mathcal{I}$ an objectspecial $\mathcal{I}$-precover of $C$ if there exists an $\mathbb{E}$-triangle $A \rightarrow X \stackrel{i}{\rightarrow} C \stackrel{\delta}{\rightarrow}$ with $A \in \mathcal{I}^{\perp_{\mathbb{E}}}$.

By a trivial morphism of $\mathbb{E}$-triangle

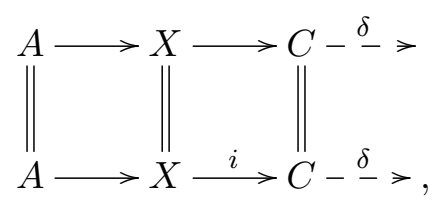

we have that any object-special $\mathcal{I}$-precover is a special $\mathcal{I}$-precover. In the following, we give a sufficient condition such that a special precovering ideal $\mathcal{I}$ is an object-special precovering ideal, that is, any object in $\mathscr{C}$ admits an object-special $\mathcal{I}$-precover.

Proposition 5.2. Let $\mathcal{I}$ be a special precovering ideal. If $\mathcal{I}^{\perp_{\mathbb{E}}}$ is an object ideal, then $\mathcal{I}$ is an object-special precovering ideal.

Proof. Let $C \in \mathscr{C}$, and take a special $\mathcal{I}$-precover $i^{\prime}: X^{\prime} \rightarrow C$ which comes from a morphism of $\mathbb{E}$-triangles

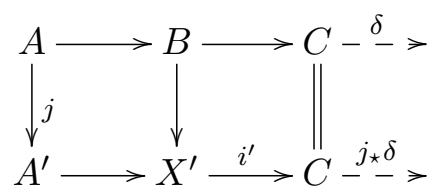

with $j \in \mathcal{I}^{\perp_{\mathbb{E}}}$. Since $\mathcal{I}^{\perp_{\mathbb{E}}}$ is an object ideal by assumption, there exist $Y \in \mathcal{I}^{\perp_{\mathbb{E}}}$ and morphisms $j_{1}: A \rightarrow Y, j_{2}: Y \rightarrow A^{\prime}$ such that $j=j_{2} j_{1}$. Then by the equality $j_{\star} \delta=j_{2 \star} j_{1_{\star}} \delta$, we can decompose the above morphism of $\mathbb{E}$-triangles to the following morphisms of $\mathbb{E}$ triangles

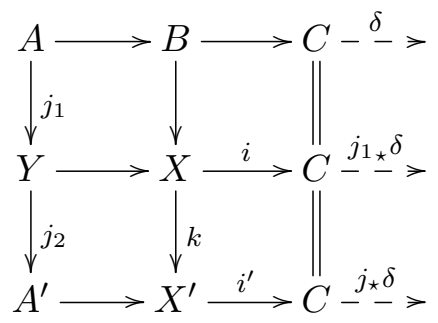

with $i=i^{\prime} k \in \mathcal{I}$. Thus $i$ is an object-special $\mathcal{I}$-precover of $C$.

In view of Proposition 5.2, it is natural to ask when the right perpendicularity of a special precovering ideal is an object ideal. To study it, we consider the following condition 
(J) Let $\mathcal{I}$ be an ideal of $\mathscr{C}$. There exists an object ideal $\mathcal{J} \subseteq \mathcal{I}^{\perp_{\mathbb{E}}}$ such that any $C \in \mathscr{C}$ admits an $\mathcal{I}$-precover $i: X \rightarrow C$ together with an $\mathbb{E}$-triangle $A \rightarrow X \stackrel{i}{\rightarrow} C \stackrel{\delta}{\rightarrow}$, where $A \in \mathcal{J}$.

Let $\mathcal{X}$ and $\mathcal{Y}$ be two classes of objects in $\mathscr{C}$. We write

$\mathcal{X} \diamond \mathcal{Y}:=\{Z \in \mathscr{C} \mid$ there exists an $\mathbb{E}$-triangle $X \rightarrow Z \rightarrow Y \stackrel{\delta}{\rightarrow}$ with $X \in \mathcal{X}$ and $Y \in \mathcal{Y}\}$.

Proposition 5.3. Let $(\mathscr{C}, \mathbb{E}, \mathfrak{s})$ be an extriangulated category with enough injective objects and $\mathcal{I}$ be a special precovering ideal of $\mathscr{C}$. The condition $(\mathrm{J})$ is satisfied if and only if $\mathcal{I}^{\perp_{\mathbb{E}}}$ is an object ideal; in this case, we have

$$
\mathcal{I}^{\perp_{\mathbb{E}}}=\langle\mathrm{Ob}(\mathcal{J}) \diamond \mathrm{Ob}(\mathbb{E}-\mathbf{i n j})\rangle
$$

Proof. The sufficiency is trivial. In the following, we prove the necessity.

Let $Z \in \mathrm{Ob}(\mathcal{J}) \diamond \mathrm{Ob}(\mathbb{E}$-inj $)$, that is, there exists an $\mathbb{E}$-triangle $X \rightarrow Z \rightarrow Y \stackrel{\delta}{-}$ with $X \in \mathcal{J}$ and $Y$ an $\mathbb{E}$-injective object. By assumption, we have $X \in \mathcal{I}^{\perp_{\mathbb{E}}}$, and hence $Z \in \mathcal{I}^{\perp_{\mathbb{E}}}$ by Lemma 4.3 . This shows that $\langle\mathrm{Ob}(\mathcal{J}) \diamond \mathrm{Ob}(\mathbb{E}$-inj $)\rangle \subseteq \mathcal{I}^{\perp_{\mathbb{E}}}$.

Conversely, let $A \in \mathscr{C}$. Then there exists an $\mathbb{E}$-triangle $A \rightarrow E \stackrel{e}{\rightarrow} C \stackrel{\delta}{\rightarrow}$ with $E$ an injective object by assumption. For the object $C$, by $(J)$ there exists an $\mathbb{E}$-triangle $K \rightarrow X \stackrel{i}{\rightarrow} C \stackrel{\gamma}{\rightarrow}$ with $K \in \mathcal{J}$ and $i: X \rightarrow C$ an $\mathcal{I}$-precover of $C$. By (ET4), we get the following commutative diagram

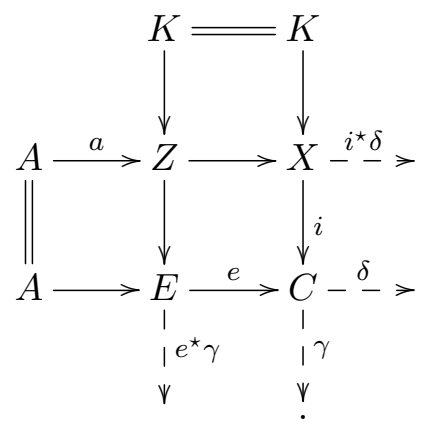

By the middle column in the above diagram, we have that $Z \in \mathrm{Ob}(\mathcal{J}) \diamond \mathrm{Ob}(\mathbb{E}$-inj). On the other hand, as in the proof of Theorem 4.4 , the morphism $a: A \rightarrow Z$ is a special $\mathcal{I}^{\perp} \mathbb{E}_{-}$ preenvelope of $A$; in particular, it is an $\mathcal{I}^{\perp_{\mathbb{E}}}$-preenvelope of $A$. Thus any $f \in \mathcal{I}^{\perp_{\mathbb{E}}}(A, B)$ factors through $a$, that is, there exists $b: Z \rightarrow B$ such that $f=b a$, which shows that $f \in\langle\mathrm{Ob}(\mathcal{J}) \diamond \mathrm{Ob}(\mathbb{E}$-inj $)\rangle$. Thus $\mathcal{I}^{\perp_{\mathbb{E}}} \subseteq\langle\mathrm{Ob}(\mathcal{J}) \diamond \mathrm{Ob}(\mathbb{E}$-inj $)\rangle$, and therefore $\mathcal{I}^{\perp_{\mathbb{E}}}=\langle\mathrm{Ob}(\mathcal{J}) \diamond$ $\mathrm{Ob}(\mathbb{E}$-inj $)\rangle$; in particular, $\mathcal{I}^{\perp_{\mathbb{E}}}$ is an object ideal.

By Propositions 5.2 and 5.3 , we immediately have the following 
Corollary 5.4. Let $(\mathscr{C}, \mathbb{E}, \mathfrak{s})$ be an extriangulated category with enough injective objects. If the condition $(\mathrm{J})$ is satisfied, then any special precovering ideal of $\mathscr{C}$ is an object-special precovering ideal.

The additive subfunctor $\mathbb{F}$ of $\mathbb{E}$ is said to have enough special injective objects if for any $A \in \mathscr{C}$, there exists an $\mathbb{F}$-triangle $A \stackrel{e}{\longrightarrow} B \longrightarrow C \stackrel{\delta}{\rightarrow}$ with $B \in \mathbb{F}$-inj, together with a morphism of $\mathbb{E}$-triangles

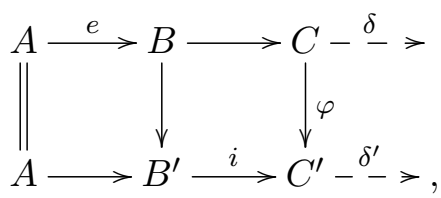

where $\varphi$ is an $\mathbb{F}$-phantom morphism.

Now we give the object-ideal's version of Theorem 4.6 compare it with 10 , Theorem 2].

Theorem 5.5. Let $(\mathscr{C}, \mathbb{E}, \mathfrak{s})$ be an extriangulated category. Then we have the following implications.

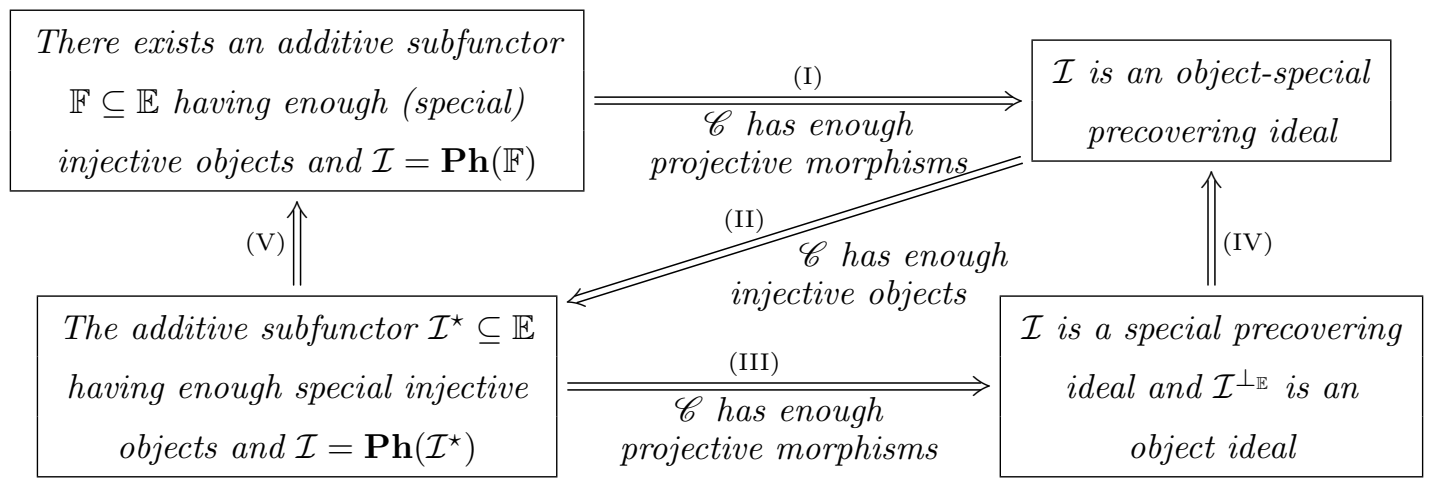

Proof. (I) For any $C \in \mathscr{C}$, there exists an $\mathbb{E}$-triangle $E \rightarrow Y \stackrel{\varphi}{\rightarrow} C \stackrel{\delta}{\rightarrow}$ with $\varphi$ an $\mathbb{F}$ phantom morphism and $E$ an $\mathbb{F}$-injective object. Because $\mathcal{I}=\mathbf{P h}(\mathbb{F})$ and $\mathbb{F}$-inj $\subseteq$ $\mathbf{P h}(\mathbb{F})^{\perp_{\mathbb{E}}}$, we have that $\varphi$ is an object-special $\mathcal{I}$-precover of $A$. Thus $\mathcal{I}$ is an object-special precovering ideal.

(II) Since $\mathcal{I}$ is an object-special precovering ideal, it is clearly an special precovering ideal, and hence $\mathcal{I}=\mathbf{P h}\left(\mathcal{I}^{\star}\right)$ by Corollary 3.10. Let $A \in \mathscr{C}$, by assumption there exists an $\mathbb{E}$-triangle $A \longrightarrow E \stackrel{e}{\longrightarrow} C \stackrel{\delta}{\rightarrow}$ with $E$ an injective object. For the object $C$, since $\mathcal{I}$ is an object-special precovering ideal, there exists an $\mathbb{E}$-triangle $K \rightarrow X \stackrel{i}{\rightarrow} C \stackrel{\gamma}{>}$ with 
$i \in \mathcal{I}$ and $K \in \mathcal{I}^{\perp_{\mathbb{E}}}$. By (ET4), we get the following commutative diagram

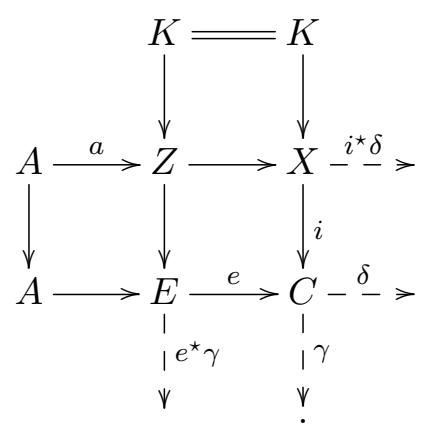

The middle row in the above diagram is an $\mathcal{I}^{\star}$-triangle. Moreover, since $K \in \mathcal{I}^{\perp_{\mathbb{E}}}$ and $E$ is an injective object, we have $Z \in \mathcal{I}^{\perp_{\mathbb{E}}}$ by Lemma 4.3. By Proposition 3.5, we have $\mathcal{I}^{\perp_{\mathbb{E}}}=\mathcal{I}^{\star}$-inj. Thus $Z \in \mathcal{I}^{\star}$-inj. Since $i \in \mathcal{I}=\mathbf{P h}\left(\mathcal{I}^{\star}\right)$, the above diagram shows that $\mathcal{I}^{\star}$ has enough special injective objects.

(III) Assume that $\mathcal{I}^{\star}$ has enough special injective objects. Of course, $\mathcal{I}^{\star}$ has enough injective morphisms, and then by Theorem $4.2, \mathcal{I}=\mathbf{P h}\left(\mathcal{I}^{\star}\right)$ is a special precovering ideal. By assumption, for any $A \in \mathscr{C}$, there exists an $\mathbb{E}$-triangle $A \stackrel{e}{\rightarrow} E \rightarrow X \stackrel{\delta}{\rightarrow}$ with $E$ an $\mathcal{I}^{\star}$-injective object, together with a morphism of $\mathbb{E}$-triangles

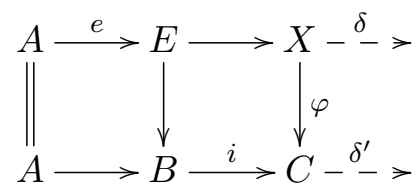

with $\varphi \in \mathbf{P h}\left(\mathcal{I}^{\star}\right)$. By Proposition 3.13 , we have $\mathbf{P h}\left(\mathcal{I}^{\star}\right)=\perp_{\mathbb{E}}\left(\mathcal{I}^{\star}\right.$-inj $)$, and hence $\varphi \in$ $\perp_{\mathbb{E}}\left(\mathcal{I}^{\star}\right.$-inj), which shows that $e$ is a special $\mathcal{I}^{\star}$-injective preenvelope of $A$.

By Proposition $3.5(2), \mathcal{I}^{\star}$-inj $=\mathcal{I}^{\perp_{\mathbb{E}}}$. So for any $a: A \rightarrow A^{\prime} \in \mathcal{I}^{\perp_{\mathbb{E}}}$, there exists $e^{\prime}: E \rightarrow A^{\prime}$ such that $a=e^{\prime} e$. This means that each morphism in $\mathcal{I}^{\perp_{\mathbb{E}}}$ factors through an $\mathcal{I}^{\star}$-injective object, and therefore $\mathcal{I}^{\perp_{\mathbb{E}}}$ is an object ideal.

(IV) By Proposition 5.2 .

(V) It is trivial.

The above theorem shows that if $(\mathscr{C}, \mathbb{E}, \mathfrak{s})$ is an extriangulated category with enough injective objects and projective morphisms, then we have the following bijective correspondence.

$$
\begin{array}{|c|c|c|}
\hline \begin{array}{c}
\text { all object-special } \\
\text { precovering ideals of } \mathscr{C}
\end{array} & \stackrel{(-)^{\star}}{\rightleftarrows} \mathbf{P h}(-) & \begin{array}{c}
\text { all additive subfunctors of } \mathbb{E} \text { having } \\
\text { enough special injective objects }
\end{array} \\
\hline
\end{array}
$$

Note that 4.2 follows from (4.1) and the morphism version of the Salce's lemma. Now, in view of (5.1), it is natural to pose the following 
Question 5.6. Does the Salce's lemma hold for object-special precovering ideals and object-special preenveloping ideals?

\section{Acknowledgments}

The first author was partially supported by Postgraduate Research and Innovation Program of Jiangsu Province (KYZZ16_0034) and Nanjing University Innovation and Creative Program for $\mathrm{PhD}$ candidate (2016011). The second author was partially supported by NSFC (11571164) and a Project Funded by the Priority Academic Program Development of Jiangsu Higher Education Institutions. The authors thank the referee for the useful suggestions.

\section{References}

[1] N. Abe and H. Nakaoka, General heart construction on a triangulated category (II): Associated homological functor, Appl. Categ. Structures 20 (2012), no. 2, 161-174.

[2] J. F. Adams and G. Walker, An example in homotopy theory, Proc. Cambridge Philos. Soc. 60 (1964), 699-700.

[3] A. A. Bẹlinson, J. Bernstein and P. Deligne, Faisceaux pervers, Astérisgue 100, Soc. Math. France, Paris, 1982.

[4] D. J. Benson, Phantom maps and purity in modular representation theory III, J. Algebra 248 (2002), no. 2, 747-754.

[5] D. J. Benson and G. Ph. Gnacadja, Phantom maps and purity in modular representation theory I, Fund. Math. 161 (1999), no. 1-2, 37-91.

[6] _ Phantom maps and purity in modular representation theory II, Algebr. Represent. Theory 4 (2001), no. 4, 395-404.

[7] A. Borel and N. Wallach, Continuous Cohomology, Discrete Subgroups, and Representations of Reductive Groups, Second editor, Mathematical Surveys and Monographs 67, American Mathematical Society, Providence, RI, 2000.

[8] S. Breaz and G.-C. Modoi, Ideal cotorsion theories in triangulated categories, arXiv:1501.06810v2.

[9] E. E. Enochs and O. M. G. Jenda, Relative Homological Algebra, De Gruyter Expositions in Mathematics 30, Walter de Gruyter, Berlin, 2000. 
[10] X. H. Fu, P. A. Guil Asensio, I. Herzog and B. Torrecillas, Ideal approximation theory, Adv. Math. 244 (2013), 750-790.

[11] A. Grothendieck, The cohomology theory of abstract algebraic varieties, in: 1960 Proc. Internat. Congress Math. (Edinburgh, 1958), 103-118, Cambridge Univ. Press, New York.

[12] D. Happel, Triangulated Categories in the Representation Theory of Finitedimensional Algebras, London Mathematical Society Lecture Note Series 119, Cambridge University Press, Cambridge, 1988.

[13] R. Hartshorne, Residues and Duality, Lecture Notes in Mathematics 20, SpringerVerlag, Berlin, Heidelberg, New York, 1966.

[14] M. Herschend, Y. Liu and H. Nakaoka, n-exangulated categories, arXiv:1709.06689v2.

[15] I. Herzog, The phantom cover of a module, Adv. Math. 215 (2007), no. 1, 220-249.

[16] N. Hoffmann and M. Spitzweck, Homological algebra with locally compact abelian groups, Adv. Math. 212 (2007), no. 2, 504-524.

[17] B. E. Johnson, Introduction to cohomology in Banach algebras, in: Algebras in Analysis, (Proceedings of the Instructional Conference and NATO Advanced Study Institute, Birmingham, 1973), 84-100, Academic Press, London, 1975.

[18] R. Kiełpiński and D. Simson, On pure homological dimension, Bull. Acad. Polon. Sci. Sér. Sci. Math. Astronom. Phys. 23 (1975), 1-6.

[19] S. Koenig and B. Zhu, From triangulated categories to abelian categories: cluster tilting in a general framework, Math. Z. 258 (2008), no. 1, 143-160.

[20] Y. Liu, Hearts of twin cotorsion pairs on exact categories, J. Algebra 394 (2013), 245-284.

[21] C. A. McGibbon, Phantom maps, in: Handbook of Algebraic Topology, 1209-1257, North-Holland, Amsterdam, 1995.

[22] D. Miličić, Lectures on Derived Categories, Preprint available at https://www.math.utah.edu/ milicic/Eprints/dercat.pdf.

[23] H. Nakaoka, General heart construction on a triangulated category (I): Unifying tstructures and cluster tilting subcategories, Appl. Categ. Structures 19 (2011), no. 6, 879-899. 
[24] H. Nakaoka and Y. Palu, Mutation via Hovey twin cotorsion pairs and model structures in extriangulated categories, arXiv:1605.05607v2.

[25] A. Neeman, The Brown representability theorem and phantomless triangulated categories, J. Algebra 151 (1992), no. 1, 118-155.

[26] D. Quillen, Higher algebraic K-theory I, in: Algebraic K-theory I: Higher K-theories, (Proceedings of the Conference, Battelle Memorial Institute, Seattle, Washington, 1972), 85-147, Lecture Notes in Math. 341, Springer, Berlin, 1973.

[27] L. Salce, Cotorsion theories for abelian groups, in: Symposia Mathematica XXIII, 11-32, Academic Press, New York, 1979.

[28] M. Schlichting, Hermitian K-theory of exact categories, J. K-Theory 5 (2010), no. 1, $105-165$.

[29] D. Simson, On pure global dimension of locally finitely presented Grothendieck categories, Fund. Math. 96 (1977), no. 2, 91-116.

[30] J.-L. Verdier, Des catégories dérivées des catégories abéliennes, Astérisque 239 (1996), 253 pp.

[31] P. Zhou and B. Zhu, Triangulated quotient categories revisited, J. Algebra, 502 (2018), 196-232.

Tiwei Zhao and Zhaoyong Huang

Department of Mathematics, Nanjing University, Nanjing 210093, Jiangsu Province, P. R. China

E-mail address: tiweizhao@hotmail.com, huangzy@nju.edu.cn 